\title{
A systematic review and meta-analysis on the prevalence of dietary supplement use by military personnel
}

\author{
Joseph J Knapik ${ }^{1,2,45^{*}}$, Ryan A Steelman ${ }^{2,4}$, Sally S Hoedebecke³, Emily K Farina ${ }^{1}$, Krista G Austin ${ }^{1,4}$
} and Harris R Lieberman ${ }^{1}$

\begin{abstract}
Background: Although a number of studies have been conducted on the prevalence of dietary supplement (DS) use in military personnel, these investigations have not been previously summarized. This article provides a systematic literature review of this topic.

Methods: Literature databases, reference lists, and other sources were searched to find studies that quantitatively examined the prevalence of DS use in uniformed military groups. Prevalence data were summarized by gender and military service. Where there were at least two investigations, meta-analysis was performed using a random model and homogeneity of the prevalence values was assessed.

Results: The prevalence of any DS use for Army, Navy, Air Force, and Marine Corps men was 55\%,60\%, 60\%, and $61 \%$, respectively; for women corresponding values were $65 \%, 71 \%, 76 \%$, and $71 \%$, respectively. Prevalence of multivitamin and/or multimineral (MVM) use for Army, Navy, Air Force, and Marine Corps men was 32\%, 46\%, 47\%, and $41 \%$, respectively; for women corresponding values were $40 \%, 55 \%, 63 \%$, and $53 \%$, respectively. Use prevalence of any individual vitamin or mineral supplement for Army, Navy, Air Force, and Marine Corps men was 18\%, 27\%, $25 \%$, and $24 \%$, respectively; for women corresponding values were $29 \%, 36 \%, 40 \%$, and $33 \%$, respectively. Men in elite military groups (Navy Special Operations, Army Rangers, and Army Special Forces) had a use prevalence of 76\% for any DS and 37\% for MVM, although individual studies were not homogenous. Among Army men, Army women, and elite military men, use prevalence of Vitamin C was 15\% for all three groups; for Vitamin E, use prevalence was $8 \%, 7 \%$, and $9 \%$, respectively; for sport drinks, use prevalence was $22 \%, 25 \%$ and $39 \%$, respectively. Use prevalence of herbal supplements was generally low compared to vitamins, minerals, and sport drinks, $\leq 5 \%$ in most investigations.
\end{abstract}

Conclusions: Compared to men, military women had a higher use prevalence of any DS and MVM. Army men and women tended to use DSs and MVM less than other service members. Elite military men appeared to use DSs and sport drinks more than other service members.

Keywords: Vitamins, Minerals, Multivitamins, Vitamin C, Vitamin E, Calcium, Iron, Protein, Creatine, Sport drink

\footnotetext{
*Correspondence: joseph.j.knapik.ctr@mail.mil

'US Army Research Institute of Environmental Medicine, Natick, MA, USA

${ }^{2}$ US Army Institute of Public Health, Aberdeen Proving Ground, MD, USA

Full list of author information is available at the end of the article
} 


\section{Background}

Dietary supplements (DSs) are commercially available products that are consumed as an addition to the usual diet. DSs include ingredients such as vitamins, minerals, herbs (botanicals), amino acids, and a variety of other substances [1]. Marketing claims made for various DSs include the ability to improve overall health status, enhance cognitive or physical performance, increase energy, promote loss of excess weight, attenuate pain, and a variety of other favorable outcomes. The Dietary Supplement Health and Education Act of 1994 [2] established the regulatory framework for DSs in the United States (US). Since this act became law, US sales of DSs have increased from \$4 billion in 1994 to $\$ 30$ billion in 2011 [3,4], an approximate 8 -fold increase over 17 years.

Patterns of DS use may differ among distinctive subpopulations. Like athletes, military personnel often have occupational tasks that require intense and prolonged periods of physical activity. Like athletes, service members may use DSs that have purported ergogenic effects to enhance their occupational performance [5-9]. Unlike athletes, service members may be working in austere and hostile surroundings under extreme environmental conditions with high risk of injury. As a result, military personnel may use DSs that purportedly enhance health or performance under these conditions. In contrast, the general US population appears to consume DSs primarily for health reasons with only minor concern for performance enhancement $[10,11]$.

This paper presents a systematic literature review describing the prevalence of DS use in military personnel. No systematic review on this topic has previously been performed. Data collected by our group suggests that the use of DSs by military personnel may exceed that of civilian populations, and that selected subgroups within the military may have even higher DS use than the general military population $[8,12]$.

\section{Methods}

Literature searches were conducted in PubMed, Ovid MEDLINE (including OLDMEDLINE), OVID Healthstar, PsycINFO, Cumulative Index to Nursing and Allied Health Literature (CINAHL), the Defense Technical Information Center (DTIC), and publications from the National Institute of Medicine. No limitations were placed on the dates of the searches with the final search completed in January 2014. To assure that descriptors were all inclusive, we examined Medical Subject Headings for "military personnel" and "DSs" in PubMed. Largely as a result of this examination, keywords selected for the search included military personnel, soldier, sailor, airmen, marine, armed forces personnel, coast guard, submariners, Navy, and Air Force personnel combined with nutrition, DS, supplement, vitamin, mineral, amino acid, protein, herb, herbal, sport drink, sport bar, nutriceuticals, neutraceuticals, food supplements, and food supplementation. To find additional studies, the reference lists of the articles obtained were searched as was the literature database of an investigator with extensive experience with DSs. In several cases, authors were contacted to obtain information that was not included in the article.

Articles were selected for the review if they were:

- Written in English,

- Provided a quantitative assessment of the prevalence of DS use or prevalence could be calculated from data in the article, and

- Participants were military personnel.

Studies were exclude if:

- Participants were other than military personnel,

- The study that did not allow separation of military personnel from others in the study,

- Prevalence could not be calculated as a percent of the total sample in the study, or

- The study that did not include specific DSs.

Data in which DSs were described by terms like "antioxidant", "pro-performance”, "herbal supplement”, "ergogenics", "thermogenics", "bodybuilding", and the like, were not included in this review because the type of DS was not specific. Exceptions were general categories of vitamins, minerals, sport drinks, sport bars, and energy drinks which were included because so many studies reported these.

Titles were first examined and abstracts were reviewed if the article appeared to involve military personnel and either nutrition or DSs. The full text of the article was retrieved if there was a possibility that DSs were included within the investigation. Quantitative prevalence data were obtained from the text of the article, from tables, or from graphs. If the data was in graphic form, prevalence was estimated from the vertical axis of the graph. Where multiple publications were found on a single study, all individual DS prevalences reported in any of the reports were included in the data extraction. The prevalence of a single type of DS reported in multiple publications from a single study was considered only once in the data extraction and analysis.

The methodological quality of the investigations was assessed using the technique of Loney et al. [13], which was developed specifically for rating prevalence investigations. Studies were graded on an 8-point scale which included assessments for sampling methods, sampling frame, sample size, measurement tools, measurement bias, response rate, statistical presentation, and description of subject sample. The 8 items were rated as either "yes" (1 point) or "no" (no point), based on specific criteria. Thus, the maximum possible score was 8 . Three authors 
independently rated each of the selected articles. Following the independent evaluations, the reviewers met to examine the scores and to reconcile major differences. The average score of the three reviewers served as the methodological quality score. Scores were converted to a percent by dividing the average score for each study by 8 and multiplying by $100 \%$.

The summary statistic derived from each study was the prevalence of specific DS use. This was calculated as the $\Sigma$ of individuals using the supplement $/ \Sigma$ of the entire sample $\times 100 \%$. This expressed use prevalence as a percent of the sample. Data in some studies required recalculation because authors expressed the data as a percent of DS users rather than as a percent of the total sample. Tables were constructed, one containing methodology of each study, and the other containing the prevalences of the DSs reported in the studies. In the methodological table, the "response rate" was calculated as the subjects whose data were used in the investigation divided by the total number of subjects who were asked to participate. The response rates reported by some authors had to be recalculated because the authors reported the number of responses received without considering data that was discarded (e.g., incomplete or improperly completed questionnaires). The prevalence table included columns for the most commonly reported DSs in all articles. These included "any" DS, any vitamins or minerals, multivitamins/multiminerals (MVM), specific vitamins and minerals, creatine, proteins, amino acids, and specific herbal supplements. In cases where a specific supplement was not discussed in a study, the space in the prevalence table was left blank. In studies where at least $4 \%$ of the sample used a particular DS not contained in the table columns, that DS was listed in the last column of the prevalence table. Where possible, studies were separated by sex and specific military subgroups (e.g., Army, Navy, Air Force, Marine Corps, Army Rangers, Army Special Forces, Navy Special Operations). In a number of cases the study authors had not separated the data into these categories and so the data was presented as combined. Data were compiled by year to examine if any temporal trends could be discerned.

The Comprehensive Meta-Analysis Statistical Package, Version 2 (Biostat, Englewood NJ). was used to perform meta-analysis on specific groups and specific DSs where there were at least two studies and where subjects were asked about "current" DS use or use $\geq 1$ time/week. A random model was used that involved providing the number of service members using the DS and those not using the DS to produce a summary prevalence estimate (SPE) with a summary 95\% confidence interval $(\mathrm{S} 95 \% \mathrm{CI})$ that represented the pooled results from the individual investigations. Homogeneity of the prevalence estimates from the studies was assessed using the Q statistic. To examine possible temporal trends, the prevalence of DSs reported in at least 3 studies were plotted by publication year. Curve fitting procedures were applied to these plots including linear, logarithmic, and second order polynomial fits.

\section{Results}

The search produced 2,930 potential publications. Figure 1 shows the number of publications included and excluded at each stage of the literature search. Thirty-three unique investigations in 38 publications met the review criteria. Seven reports were in government technical reports [14-20], two were only in abstract form [21,22], 9 were in an Institute of Medicine report on the use of DSs in military personnel [23-31], and 20 reports were in peerreviewed journal articles [5-9,12,32-45]. Three individual studies had two reports each $[15,19,25,30,32,38]$ and one produced three relevant publications $[8,41,42]$.

Two studies that involved DS use in military groups were excluded. One study of repatriated Vietnam prisoners-ofwar was not included because of the unusual circumstances and because the vitamin intake was not voluntary in some cases [46]. Also excluded was one study that asked physicians about DSs reported by patients, as opposed to participants self-reporting their DS use [47].

Table 1 shows the subjects and methods used to obtain data from the 33 unique investigations. Most studies involved US service members, but two studies were conducted among deployed British soldiers [7,40] and one in Macedonian Special Operations Soldiers [45]. Among the US service members, several studies examined elite military service members including Army Rangers [5,22,27,30,38], Army Special Forces [12,28], and Navy Sea, Air, Land (SEAL) personnel [36]. Other studies involved general samples of Army soldiers $[6,8,14,16,23,26]$, Air Force personnel [17,31], Navy [37] and Marine Corps personnel [9,18,37], Marine and Air Force trainees [20,36,39], senior military officers [29], military officers in training [15,35], men initiating training to become Ranger and Special Forces soldiers [33], and multiple service groups $[19,24,25,34,43,44]$.

In most unique studies $(\mathrm{n}=31)$, the data were collected by having service members self-report their DS use on questionnaires. Most of the questionnaires were specifically designed to obtain information on DSs and focused on this topic [6-9,12,17,21-24,26-31,33,34,36,38-42,45]. Other studies obtained DS information from questionnaires that had items on DS use but were designed for more general purposes, often to collect a range of nutritional measures $[5,14,16,18-20,25,37,43,44]$. Two unique investigations obtained DS information from 7-day food records complimented with interviews by dietitians $[15,32,35]$.

The reporting timeframe differed among studies. In many investigations, service members reported DS use $\geq 1$ time/ week, or these data could be calculated from the information provided in the articles $[6,8,16,19,25-29,33,41,42]$. Other studies examined current use but did not clearly define 


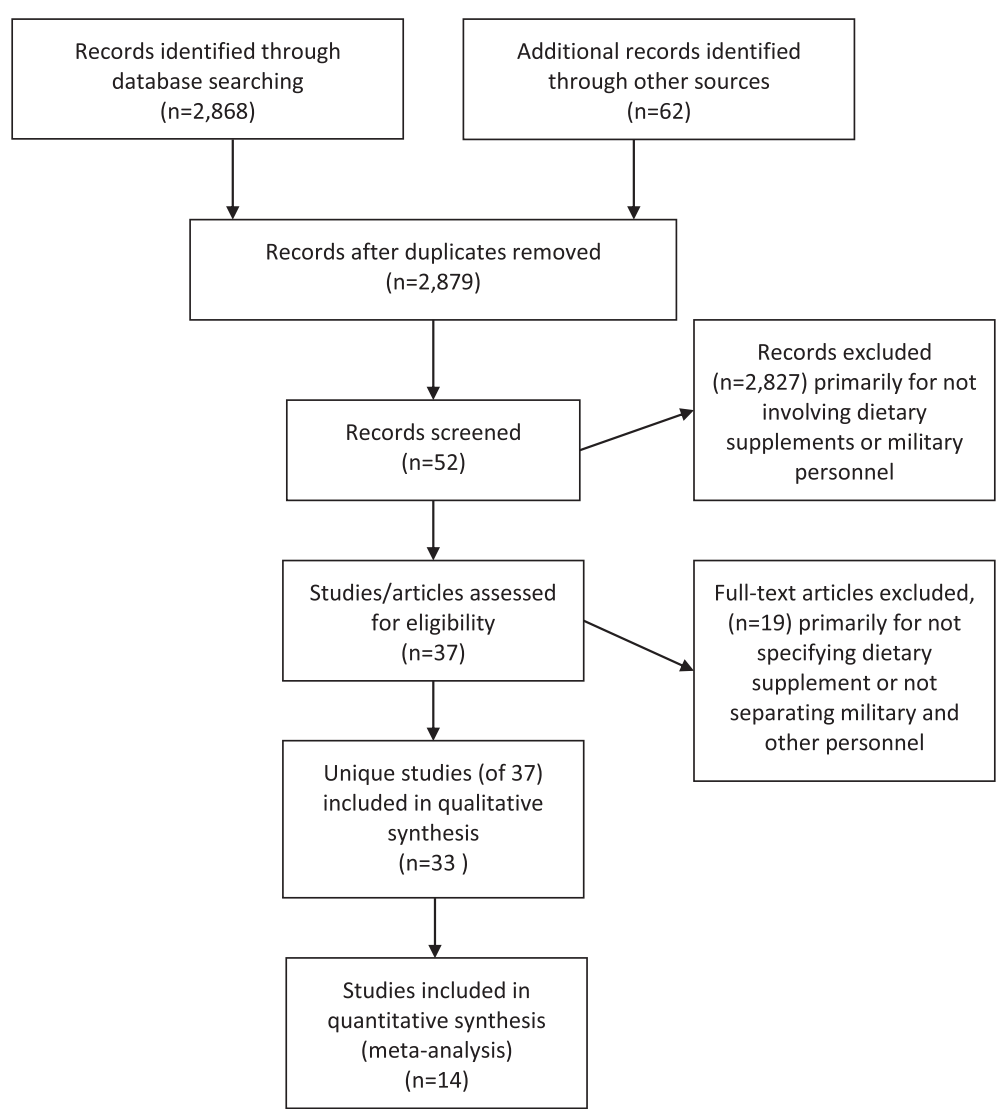

Figure 1 Records included and excluded at each stage of literature review.

the frequency of use $[7,12,14,23,24,30,31,34,38,40]$. In other studies, service members reported use in the last 7 days [15,32,35], last month [9], last 3 months [24,45], last 6 months [22], last 12 months [18,37,43], or last two years [17]. Two studies examined the lifetime prevalence of DS use in Marine and Air Force basic trainees [36,39]. Two studies reported daily use $[5,44]$ and in one study the reporting timeframe was not stated [21].

The response rate was not provided in 15 unique studies [5,9,12,15,16,20-23,27-29,31,35,45]. In the 18 other unique investigations, the response rates ranged from $35 \%$ [37] to $99 \%$ [33] with only 10 unique investigations having response rates $\geq 66 \%[7,8,14,18,26,33,34,36,43,44]$.

Rating from the methodological quality reviews ranged from $25 \%$ to $91 \%$ of available points, with an average \pm standard deviation rating of $52 \pm 20 \%$. Only 8 studies $(24 \%)$ had a rating of $70 \%$ or better. Several studies were only reported in an Institute of Medicine publication on DS use in the military [48] and received relatively lower scores because of the lack of detail provided [23,24,26-29,31]. The study by Bray et al. [19] was particularly well conducted in that the investigators attempted to collect a random sample of the entire military and clearly outlined their sampling methods, sampling frame, questionnaire, and results.
Table 2 shows available data on the prevalence of DS use by service members. Twenty-six unique investigations provided the prevalence of "any" DS use [5-9,12,15,18-22, $24,26-29,31,33,35,36,38-40,43,45]$ and 17 reported on multivitamin use [6-9,12,16,19,21-24,26-29,33,45]. Only 10 unique investigations reported on specific vitamins and mineral supplements [6,12,22-24,26,28,29,33,45] and 7 reported on specific herbal supplements $[6,17,23,29$, $33,39,45]$. Nineteen unique investigations reported on supplementation with creatine [5-7,12,18,21-24,26-28,33,34, $36,38-40,45]$ and 20 reported on amino acid and/or protein supplements [5-9,12,16,21-24,26-28,33,34,38-40,45]. Finally, supplementation with sport drinks were reported in 11 investigations [5,8,12,21,23,26-29,39,45], sport bars in 10 studies [8,12,16,21-23,26-28,33], and energy drinks in 3 investigations $[8,9,44]$. None of the studies identified the use prevalence of specific brands of MVM, vitamins, minerals, amino acids, proteins, botanicals, sport drinks or sport bars.

Table 3 shows summary data on US military DS use where questionnaires had asked about "current use" or use $\geq 1$ time per week. Only 13 studies were included in these meta-analyses (Figure 1) because the other studies used different reporting timeframes or reported on a DS 
Table 1 Methods in investigations of DS use by military personnel

\begin{tabular}{|c|c|c|c|c|c|}
\hline Study & Subjects & $\begin{array}{l}\text { Methods for collecting } \\
\text { supplement information }\end{array}$ & Reporting timeframe & $\begin{array}{l}\text { Response rate (\%) - proportion } \\
\text { of population participating } \\
\text { in investigation }\end{array}$ & $\begin{array}{l}\text { Methodological } \\
\text { quality score (\%) }\end{array}$ \\
\hline Carlson et al. [14] & $\begin{array}{l}43 \text { ô US Army senior } \\
\text { non-commissioned officers }\end{array}$ & Questionnaire with items on DS use & Current Use & 86 & 75 \\
\hline Klicka et al. [15] & $\begin{array}{l}119 \AA \& 86 \text { \& cadets at the } \\
\text { US Military Academy at } \\
\text { West Point NY }\end{array}$ & 7-day dietary record with interview & Use over 7 days & Not provided & 38 \\
\hline Schneider et al. [21] & 91 đ US Navy SEAL personnel & Questionnaire focused on DS use & Current Use & Not provided & 25 \\
\hline Kennedy and Arsenault [33] & $\begin{array}{l}\text { 2,215 ô US Army soldiers entering } \\
\text { Special Forces and Ranger training }\end{array}$ & Questionnaire focused on DS use & $\begin{array}{l}\text { Current use, } \geq 1 \text { time/wk, } \\
\text { within last } 3 \text { months }\end{array}$ & 99 & 66 \\
\hline Warber et al. [16] & $\begin{array}{l}\text { 2,547 ô \& } 494 \text { o US Army } \\
\text { soldiers from } 32 \text { Army } \\
\text { installations world-wide }\end{array}$ & Questionnaires with items on DSs & Current use $\geq 1$ time/wk & Not provided & 59 \\
\hline McGraw et al. [22] & 367 đ US Army Rangers & Questionnaire focused on DS use & Use in past 6 months & Not provided & 41 \\
\hline Sheppard et al. [34] & $\begin{array}{l}102 \text { đ \& } 31 \text { q US service } \\
\text { members using health clubs }\end{array}$ & $\begin{array}{l}\text { Questionnaire focused on creatine } \\
\text { and use of other DSs }\end{array}$ & Current use & 70 & 66 \\
\hline Arsenault \& Cline [35] & 50 q officers in Basic Officer Training & 7-day dietary record with interview & Use over 7 day period & Not provided & 42 \\
\hline Stevens and Olsen [36] & $\begin{array}{l}439 \AA \& \text { \& } 60 \text { q US marine } \\
\text { or AF basic trainees }\end{array}$ & $\begin{array}{l}\text { Questionnaire focused on } \\
\text { ergogenic DS use }\end{array}$ & Any use in lifetime & 91 & 91 \\
\hline Shanks [17] & $224 q$ active duty US AF women & $\begin{array}{l}\text { Questionnaire focused on herbal } \\
\text { therapy }\end{array}$ & Use in the last 2 years & 45 & 75 \\
\hline Bovill et al. [12] & 119 o US Army Special Forces soldiers & Questionnaire on nutrition and DS use & Current use & Not provided & 29 \\
\hline Deuster et al. [5] & $\begin{array}{l}38 \text { o US Army Rangers at } \\
\text { Fort Bragg, North Carolina }\end{array}$ & $\begin{array}{l}\text { General health questionnaire } \\
\text { with items on DSs }\end{array}$ & Daily Use & Not provided & 29 \\
\hline Brasfield [6] & $\begin{array}{l}750 \text { o \& } 124 \text { q US Army soldiers } \\
\text { from } 16 \text { Army posts }\end{array}$ & Questionnaire focused on DS use & Current use $\geq 1$ time/wk & 58 & 59 \\
\hline Castillo et al. [18] & $\begin{array}{l}1,326 \text { ô \& } 120 \text { o US marines } \\
\text { at Camp Pendleton, California }\end{array}$ & $\begin{array}{l}\text { Questionnaire (Marine Health } \\
\text { Behavior Survey) with items on DSs }\end{array}$ & Use in last year & 85 & 59 \\
\hline Bray et al. [19,25] & $\begin{array}{l}12,119 \text { o \& } 4,027 \text { q US quad-service } \\
\text { members (23\% Army; } 29 \% \text { Navy, } \\
21 \% \text { Marine Corps, } 28 \% \text { AF) }\end{array}$ & Questionnaire with items on DS use & $\begin{array}{l}\text { Current use } \geq 1 \text { time/wk } \\
\text { in last year }\end{array}$ & 53 & 88 \\
\hline Smith et al. [37] & $\begin{array}{l}1,009 \text { of and } 296 \text { \& US Navy and } \\
\text { Marine Corps personnel (72\% Navy, } \\
22 \% \text { Marine Corps, } 6 \% \\
\text { Navy Reserve/Guard) }\end{array}$ & Questionnaire with items on DS use & Use in past year & 35 & 79 \\
\hline Johnson et al. [30,38] & 294 đ US Army Rangers & Questionnaire focused on DS use & Current Use & 39 & 41 \\
\hline Corum [23] & 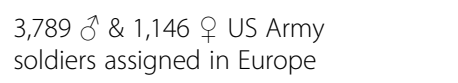 & Questionnaire focused on DS use & Current use & Not provided & 38 \\
\hline French [24] & $\begin{array}{l}376 \text { US service members, active duty, } \\
\text { National Guard, or reserves }\end{array}$ & $\begin{array}{l}\text { On-line questionnaire } \\
\text { focused on DS use }\end{array}$ & Use in past 3 months & 60 & 38 \\
\hline
\end{tabular}


Table 1 Methods in investigations of DS use by military personnel (Continued)

\begin{tabular}{|c|c|c|c|c|c|}
\hline Lieberman [29] & $\begin{array}{l}284 \lesssim \& 31 \text { o US senior military officers } \\
\text { at the US Army War College, all services }\end{array}$ & Questionnaire focused on DS use & Use $\geq 1$ time/wk & Not provided & 34 \\
\hline Lieberman [27] & 768 ठै US Army Rangers & Questionnaire focused on DS use & Use $\geq 1$ time/wk & Not provided & 25 \\
\hline Lieberman [28] & 152 o US Army Special Forces soldiers & Questionnaire focused on DS use & Use $\geq 1$ time/wk & Not provided & 29 \\
\hline Lieberman [26] & 444 ô \& 40 ㅇ US Army soldiers & Questionnaire focused on DS use & Use $\geq 1$ time/wk & 80 & 46 \\
\hline Thomasos 2008 [31] & $\begin{array}{l}10,985 \text { US AF personnel at } 27 \\
\text { major installations }\end{array}$ & Questionnaire focused on DS use & Current use & Not provided & 38 \\
\hline Young and Stephens [39] & $\begin{array}{l}236 \hat{~} \hat{\alpha} \& 83 \text { o } \text { US Marine Corps } \\
\text { recruits entering basic training }\end{array}$ & Questionnaire focused on DS use & Use at any time in past & 65 & 54 \\
\hline Boos et al. [7] & $\begin{array}{l}889 \text { ô \& } 128 \text { o UK Soldiers } \\
\text { deployed in Basra, Iraq }\end{array}$ & Questionnaire focused on DS use & Current Use & 66 & 66 \\
\hline Lieberman et al. [8,41,42] & $\begin{array}{l}859 \text { O \&131 q US Army soldiers from } \\
11 \text { locations including two overseas; } \\
17 \text { Special Forces soldiers }\end{array}$ & Questionnaire focused on DS use & $\begin{array}{l}\text { Use } \geq 1 \text { time/wk in last } \\
6 \text { months }\end{array}$ & $\sim 80$ & 71 \\
\hline Wells \& Webb [20] & $\begin{array}{l}197 \text { US AF trainees in Tactical } \\
\text { Air Control Party training }\end{array}$ & Questionnaire with items on DSs & Current Use & Not provided & 41 \\
\hline Boos et al. [40] & $\begin{array}{l}78 \text { o \& } 9 \text { q UK soldiers deployed } \\
\text { in Afghanistan }\end{array}$ & Questionnaire focused on DS use & Current use & 58 & 29 \\
\hline Jacobson et al. [43] & $\begin{array}{l}72,718 \text { of \& } 33,980 \text { o US service members } \\
\text { (active \& reserve) enrolled in the } \\
\text { Millennium Cohort Study }\end{array}$ & $\begin{array}{l}\text { Questionnaire including item } \\
\text { on DS use }\end{array}$ & Use in last 12 months & 68 & 88 \\
\hline Toblin et al. [44] & $\begin{array}{l}988 \text { o US Army soldiers and } \\
\text { marines deployed to Afghanistan }\end{array}$ & $\begin{array}{l}\text { Questionnaire including item } \\
\text { on energy drinks }\end{array}$ & Daily Use & 79 & 71 \\
\hline Kjertakov et al. 2013 [45] & $\begin{array}{l}132{ }^{\Uparrow} \text { Macedonian Special } \\
\text { Operations soldiers }\end{array}$ & Questionnaire focused on DS use & Use in last 3 months & Not provided & 41 \\
\hline Cassler et al. [9] & $\begin{array}{l}310 \text { o \& } 19 \text { o US marines deployed } \\
\text { in Afghanistan (Camp Leatherneck) }\end{array}$ & Questionnaire focused on DS use & Use in last 30 days & Not provided & 38 \\
\hline
\end{tabular}




\begin{tabular}{|c|c|c|c|c|c|c|c|c|c|c|c|c|c|c|c|c|c|c|c|}
\hline \multirow[t]{2}{*}{ Study } & \multirow[t]{2}{*}{ Subjects } & \multicolumn{18}{|c|}{ Proportion of entire sample reporting use (\%) } \\
\hline & & $\begin{array}{l}\text { Any DS } \\
\text { Use }\end{array}$ & $\begin{array}{l}\text { Any Vit (V), } \\
\text { Minl (M), or } \\
\text { Vit/Minl } \\
\text { (VM) Suppl }\end{array}$ & $\begin{array}{l}\text { MV or } \\
\text { MVM } \\
\text { Suppl }\end{array}$ & $\begin{array}{l}\text { Vit } \\
\text { A }\end{array}$ & $\begin{array}{l}\text { Vit B } \\
\text { or B } \\
\text { Cmpx }\end{array}$ & $\begin{array}{l}\text { Vit } \\
\text { C }\end{array}$ & $\begin{array}{l}\text { Vit } \\
\text { D }\end{array}$ & $\begin{array}{l}\text { Vit } \\
\text { E }\end{array}$ & $\mathrm{Fe}$ & $\mathrm{Ca}$ & $\mathrm{Zn}$ & Creatine & $\begin{array}{l}\text { Amino acids } \\
\text { (A), Prot (P), or } \\
\text { Amino acids \& } \\
\text { Prot (AP) }\end{array}$ & $\begin{array}{l}\text { Ginkgo } \\
\text { Biloba }\end{array}$ & Ginseng & $\begin{array}{l}\text { Sport } \\
\text { drink }\end{array}$ & $\begin{array}{l}\text { Sport } \\
\text { bar }\end{array}$ & $\begin{array}{l}\text { Other DSs } \\
\text { in study }\end{array}$ \\
\hline Carlson et al. [14] & $\begin{array}{l}43 \text { đ US Army senior } \\
\text { non-commissioned } \\
\text { officers }\end{array}$ & & VM-7 & & & & & & & & & & & & & & & & \\
\hline \multirow[t]{2}{*}{ Klicka et al. [15,32] } & 119 ô cadets at USMA & 14 & & & & & & & & & & & & & & & & & \\
\hline & $86 q$ cadets at USMA & 35 & & & & & & & & & & & & & & & & & \\
\hline Schneider et al. [21] & 91 đ̊ SEAL personnel & 78 & & 26 & & & & & & & & & 32 & AP-12 & & & 19 & 40 & \\
\hline Kennedy \& Arsenault [33] & $\begin{array}{l}2,215 \text { o US Army } \\
\text { Special Forces and } \\
\text { Rangers in training }\end{array}$ & 64 & & 30 & $5^{b}$ & 3 & 13 & & 6 & 3 & 5 & 3 & 15 & $A-9^{a}$ & & 5 & & 11 & $\mathrm{Cr}-4$ \\
\hline \multirow[t]{2}{*}{ Warber et al. [16] } & $\begin{array}{l}2,547 \text { ô US } \\
\text { Army Soldiers }\end{array}$ & & V-18 & 24 & & & & & & & & & & $A-10$ & & & & 8 & \\
\hline & $\begin{array}{l}494 \text { q U US } \\
\text { Army Soldiers }\end{array}$ & & V-24 & 35 & & & & & & & & & & $A-4$ & & & & 3 & \\
\hline McGraw et al. [22] & $\begin{array}{l}367 \text { đ̃ US } \\
\text { Army Rangers }\end{array}$ & 36 & & 16 & & & 7 & & & & & & 19 & AP-14 & & & & 6 & \\
\hline Sheppard et al. [34] & $\begin{array}{l}102 \text { o \& } 31 \text { q } \\
\text { US military health } \\
\text { clubs users }\end{array}$ & & V-65 M-47 & & & & & & & & & & 29 & P-45 & & & & & Caf-32 \\
\hline Arsenault \& Cline [35] & $\begin{array}{l}50 \text { \& US officers in } \\
\text { Basic Officer Training }\end{array}$ & 38 & & & & & & & & & & & & & & & & & \\
\hline Stevens \& Olsen [36] & $\begin{array}{l}439 \text { đ \& } 60 \text { o US } \\
\text { Marine Corps or AF } \\
\text { basic trainees }\end{array}$ & 41 & & & & & & & & & & & 23 & & & & & & AD-8 \\
\hline Shanks [17] & $\begin{array}{l}224 \text { q US active } \\
\text { duty AF women }\end{array}$ & & & & & & & & & & & & & & 5 & 5 & & & $\begin{array}{l}\text { Eph-5; Gar-4; } \\
\text { Ech-5; SJW-5 }\end{array}$ \\
\hline Bovill et al. [12] & $\begin{array}{l}119 \text { o US Army Special } \\
\text { Forces soldiers }\end{array}$ & 90 & & 55 & & & 20 & & 12 & & 9 & & 18 & $P-24$ & & & 71 & 52 & \\
\hline Deuster et al. [5] & 38 đ US Army Rangers & 82 & & & & & & & & & & & 13 & $P-24$ & & & 82 & & Eph-13 \\
\hline \multirow[t]{2}{*}{ Brasfield [6] } & 750 ô US Army soldiers & 59 & & 33 & 8 & $7^{c}$ & 17 & & 10 & 7 & 12 & 3 & 16 & $A-6$ & 6 & 14 & & & \multirow{2}{*}{$\begin{array}{l}\text { K-7; Vit B } \text { B }_{6} \text {; } \\
\text { CP-4; Eph-13; } \\
\text { Gar-7; Ech-4 }\end{array}$} \\
\hline & 124 q US Army soldiers & 70 & & 40 & 7 & & 15 & & 7 & 12 & 14 & & & & & 6 & & & \\
\hline Castillo et al. [18] & $\begin{array}{l}1,326 \text { o \& } 120 \text { क } \\
\text { US marines }\end{array}$ & 54 & VM-13 & & & & & & & & & & 18 & & & & & & $\begin{array}{l}\text { Eph-24; } \\
\text { AD-5 }\end{array}$ \\
\hline
\end{tabular}


Table 2 Prevalence of dietary supplement use by military personnel (Continued)

\begin{tabular}{|c|c|c|c|c|c|c|c|c|c|c|c|c|c|c|c|c|c|c|}
\hline \multirow[t]{10}{*}{ Bray et al. [19] } & $\begin{array}{l}12,119 \text { o US } \\
\text { quad-service members }\end{array}$ & 58 & VM-25 & 43 & & & & & & & & & & & & & & \\
\hline & $\begin{array}{l}4,027 \text { q US quad-service } \\
\text { members }\end{array}$ & 71 & VM-37 & 56 & & & & & & & & & & & & & & \\
\hline & 2,818 đ̂ Army Soldiers & 55 & VM-24 & 38 & & & & & & & & & & & & & & \\
\hline & 821 o Army Soldiers & 66 & VM-34 & 50 & & & & & & & & & & & & & & \\
\hline & 3,341 § Navy Sailors & 60 & VM-27 & 46 & & & & & & & & & & & & & & \\
\hline & 1,286 + Navy Sailors & 71 & VM-36 & 55 & & & & & & & & & & & & & & \\
\hline & 2,767 ð̊ Marines & 61 & VM-24 & 41 & & & & & & & & & & & & & & \\
\hline & 589 q Marines & 71 & VM-33 & 53 & & & & & & & & & & & & & & \\
\hline & 3,193 ð AF Airmen & 60 & VM-25 & 47 & & & & & & & & & & & & & & \\
\hline & 1,331 우 AF Airmen & 76 & $V M-40$ & 63 & & & & & & & & & & & & & & \\
\hline Smith et al. [37] & $\begin{array}{l}1,009 \text { of and } 296 \text { o } \\
\text { US Navy and Marine } \\
\text { Corps personnel }\end{array}$ & & $V-12$ & & & & & & & & & & & & & & & \\
\hline Johnson et al. [30,38] & $\begin{array}{l}294 \text { ð US } \\
\text { Army Rangers }\end{array}$ & 56 & & & & & & & & & & 26 & P-35 A-4 & & & & & \\
\hline Corum [23] & $\begin{array}{l}\text { 3,789 o \& 1,146 P } \\
\text { US Army Soldiers } \\
\text { assigned in Europe }\end{array}$ & & & 34 & 13 & & 24 & & & 14 & 19 & 13 & P-14 & 4 & 7 & 43 & 17 & $\begin{array}{l}\text { K-12; Ech-4; } \\
\text { Gar-5; Vit } \\
\text { B6-12; Caf-18 }\end{array}$ \\
\hline French [24] & $\begin{array}{l}376 \text { US service } \\
\text { members }\end{array}$ & 69 & & 57 & & 8 & & 3 & 9 & & 13 & 6 & P-14 A-4 & & & & & $\begin{array}{l}\Omega 3 F A-9 ; \\
\text { GlCon-7; } \\
\text { FSO-4 }\end{array}$ \\
\hline \multirow[t]{2}{*}{ Lieberman [29] } & $\begin{array}{l}284 \hat{\jmath} \text { US senior } \\
\text { military officers }\end{array}$ & 71 & & 39 & 5 & 6 & 17 & & 22 & & 5 & & & 5 & & 10 & & Gar-6 \\
\hline & $\begin{array}{l}31 \text { q US senior } \\
\text { military officers }\end{array}$ & 81 & & 52 & 16 & $36^{d}$ & 29 & & 32 & & 32 & & & & & & & $M g-13$ \\
\hline Lieberman [27] & $\begin{array}{l}768 \text { o US Army } \\
\text { Rangers }\end{array}$ & 81 & & 23 & & & & & & & & 19 & PA-18 & & & 41 & 6 & $A D-7$ \\
\hline Lieberman [28] & $\begin{array}{l}152 \text { ô US Army } \\
\text { Special Forces Soldiers }\end{array}$ & 65 & & 32 & & & 11 & & 7 & & & 16 & $P-16$ & & & 36 & 15 & $A D-6$ \\
\hline \multirow[t]{2}{*}{ Lieberman [26] } & $\begin{array}{l}444{ }^{\lambda} \text { US Army } \\
\text { Soldiers }\end{array}$ & 55 & & 30 & 5 & & 13 & 5 & 6 & & 6 & 5 & P-13 & & & 20 & 5 & \\
\hline & 40 q US Army Soldiers & 70 & & 28 & & $23^{e}$ & 13 & 8 & 8 & 10 & 15 & & P-8 & & & 28 & & \\
\hline Thomasos [31] & 10,985 US AFpersonnel & 69 & & & & & & & & & & & & & & & & \\
\hline $\begin{array}{l}\text { Young \& } \\
\text { Stephens [39] }\end{array}$ & $\begin{array}{l}236 \text { ô \& } 83 \text { o US Marine } \\
\text { Corps recruits entering } \\
\text { basic training }\end{array}$ & 50 & $V-26$ & & & & & & & & 3 & 26 & $P-43$ & 6 & & 36 & & $\begin{array}{l}\text { NO-16;Glu-16; } \\
\text { GlCon-9 }\end{array}$ \\
\hline
\end{tabular}


Table 2 Prevalence of dietary supplement use by military personnel (Continued)

\begin{tabular}{|c|c|c|c|c|c|c|c|c|c|c|c|c|c|c|c|c|}
\hline Boos et al. [7] & $\begin{array}{l}889 \text { đ \& } 128 \text { o U UK } \\
\text { Soldiers deployed } \\
\text { in Iraq }\end{array}$ & 32 & & 2 & & & & & & & 1 & P-19 A-18 & & & & Caf-4 \\
\hline \multirow[t]{3}{*}{ Lieberman et al. $[8,41,42]$} & $\begin{array}{l}859 \text { ๙ US Army } \\
\text { Soldiers }\end{array}$ & $53^{f}$ & VM-17 & 37 & & & & & & & & PA-20 & & 23 & 6 & ED-41 \\
\hline & $\begin{array}{l}131 \text { o US } \\
\text { Army Soldiers }\end{array}$ & $57^{f}$ & VM-23 & 41 & & & & & & & & PA-9 & & 24 & 4 & ED-25 \\
\hline & $\begin{array}{l}17 \text { o US Special } \\
\text { Forces Soldiers }\end{array}$ & $77^{f}$ & VM-64 & 64 & & & & & & & & PA-47 & & 32 & 7 & \\
\hline Wells \& Webb [20] & $\begin{array}{l}197 \text { US AF trainees } \\
\text { in Tactical Air Control } \\
\text { Party training }\end{array}$ & 73 & & & & & & & & & & & & & & \\
\hline Boos et al. [40] & $\begin{array}{l}78 \text { ô \& } 9 \text { q UK } \\
\text { Soldiers deployed } \\
\text { in Afghanistan }\end{array}$ & 40 & & & & & & & & & 1 & PA-34 & & & & CP-15 \\
\hline Jacobson et al. [43] & $\begin{array}{l}72,718 \text { o and } 33,980 \text { o } \\
\text { US service members } \\
\text { (active \& reserve) }\end{array}$ & 47 & & & & & & & & & & & & & & \\
\hline Toblin et al. [44] & $\begin{array}{l}988 \text { ô deployed } \\
\text { US Army soldiers } \\
\text { and marines }\end{array}$ & & & & & & & & & & & & & & & ED-45 \\
\hline \multirow[t]{2}{*}{$\begin{array}{l}\text { Kjertakov } \\
\text { et al. [45] }\end{array}$} & $\begin{array}{l}780^{\Uparrow} \text { Macedonian } \\
\text { Rangers }\end{array}$ & 64 & & 47 & 13 & 14 & 44 & 9 & 8 & 183 & 3 & $P-8 A-5$ & 0 & 15 & & $\begin{array}{l}\text { VitB }-14 ; \\
\text { Vit } B_{12}-6 ; \\
M g-10\end{array}$ \\
\hline & $\begin{array}{l}54 \text { ôn Macedonian } \\
\text { Special Forces } \\
\text { Soldiers }\end{array}$ & 70 & & 54 & 13 & 24 & 54 & 15 & 13 & 112 & 2 & P-7 A-11 ${ }^{\mathrm{a}}$ & 2 & 15 & & $\begin{array}{l}\text { VitB }{ }_{6}-13 ; \\
\text { Vit } B_{12}-9 ; \\
\text { Mg-16; } \\
\text { Se-4; Glu-4 }\end{array}$ \\
\hline \multirow[t]{2}{*}{ Cassler et al. [9] } & $\begin{array}{l}310 \text { o US marines } \\
\text { deployed in } \\
\text { Afghanistan }\end{array}$ & 72 & & 47 & & & & & & & & P-64 & & & & ED-42 \\
\hline & $\begin{array}{l}19 \text { o US marines } \\
\text { deployed in } \\
\text { Afghanistan }\end{array}$ & 42 & & & & & & & & & & & & & & \\
\hline
\end{tabular}

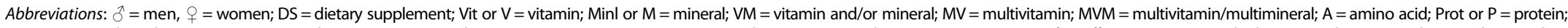
$\mathrm{AP}=$ amino acids and protein; $\mathrm{cmpx}=$ complex; $\mathrm{Fe}=$ iron; $\mathrm{Ca}=$ calcium; $\mathrm{Zn}=$ zinc; $\mathrm{Mg}=$ magnesium; $\mathrm{Cr}=$ chromium; $\mathrm{Se}=$ selenium; $\mathrm{K}=$ potassium; $\mathrm{Caf}=\mathrm{caffeine} ; \mathrm{ED}=$ energy drink; $\mathrm{Gar}=$ garlic; $\mathrm{AD}=$ androstenedione; $\mathrm{Eph}=$ ephedrine; FSO = flax seed oil; Glu = glutamine; Ech-echinacea; SJW = Saint John's Wort; $\Omega 3 F A=$ omega-3-fatty acid; GICon = glucosamine chondroitin; NO = nitric acid; CP = chromium picolinate; AF = Air Force; SEAL = Sea, Air, Land (US Navy special operations personnel); UK = United Kingdom; USMA = United State Military Academy.

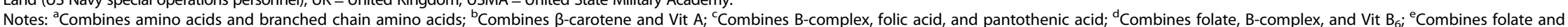
Vit $B_{6} ;$ Excludes sport drinks/bars/gels. 
Table 3 Summary data on prevalence of dietary supplement use by military personnel by gender and service

\begin{tabular}{|c|c|c|c|c|c|c|}
\hline $\begin{array}{l}\text { Dietary } \\
\text { supplement }\end{array}$ & Group & $\begin{array}{c}\text { Studies } \\
\text { (reference number) }\end{array}$ & $\begin{array}{c}\text { Individual study } \\
\text { prevalence, mean } \\
(95 \% \mathrm{Cl}) \\
(\%)\end{array}$ & $\begin{array}{l}\text { Total sample } \\
\text { size (n) }\end{array}$ & $\begin{array}{l}\text { Summary prevalence } \\
\text { estimate }(95 \% \mathrm{Cl})(\%)\end{array}$ & $\begin{array}{c}\text { Homogeneity of } \\
\text { summary prevalence } \\
\text { estimate } \\
\text { (Q Statistic p-value) }\end{array}$ \\
\hline \multirow[t]{23}{*}{ Any } & \multirow[t]{4}{*}{ Army Men } & Brasfield [6] & $59(56-63)$ & 4,871 & $55(53-56)$ & 0.77 \\
\hline & & Bray et al. [19] & $55(53-57)$ & & & \\
\hline & & Lieberman [26] & $55(50-60)$ & & & \\
\hline & & Lieberman et al. [8] & $53(50-56)$ & & & \\
\hline & Navy Men & Bray et al. [19] & $60(58-62)$ & 3,341 & & \\
\hline & AF Men & Bray et al. [19] & $60(58-62)$ & 3,193 & & \\
\hline & Marine Men & Bray et al. [19] & $61(59-63)$ & 2,767 & & \\
\hline & \multirow[t]{4}{*}{ Army Women } & Brasfield [6] & $70(62-78)$ & 1,844 & $65(60-70)$ & 0.14 \\
\hline & & Bray et al. [19] & $66(63-69)$ & & & \\
\hline & & Lieberman [26] & $70(56-84)$ & & & \\
\hline & & Lieberman et al. [8] & $57(49-66)$ & & & \\
\hline & Navy Women & Bray et al. [19] & $71(68-74)$ & 1,286 & & \\
\hline & AF Women & Bray et al. [19] & $76(74-78)$ & 1.331 & & \\
\hline & Marine Women & Bray et al. [19] & $71(67-75)$ & 589 & & \\
\hline & \multirow{2}{*}{$\begin{array}{l}\text { Army Officers in } \\
\text { Training, Women }\end{array}$} & Klicka et al. [15] & $35(25-45)$ & 136 & $36(28-44)$ & 0.72 \\
\hline & & Arsenault et al. [35] & $38(25-51)$ & & & \\
\hline & Navy SEALS & Schneider et al. [21] & $78(70-87)$ & 1,479 & $76(65-85)$ & $<0.01$ \\
\hline & Army SF & Bovill et al. [12] & $90(85-95)$ & & & \\
\hline & Army Rangers & Deuster et al. [5] & $82(70-94)$ & & & \\
\hline & Army Rangers & Johnson et al. [38] & $56(50-62)$ & & & \\
\hline & Army Rangers & Lieberman [27] & $81(78-84)$ & & & \\
\hline & Army SF & Lieberman [28] & $65(57-73)$ & & & \\
\hline & Army SF & Lieberman et al. [8] & 77 (57-97) & & & \\
\hline \multirow[t]{19}{*}{ Multivitamin } & \multirow[t]{5}{*}{ Army Men } & Warber et al. [16] & $24(22-26)$ & 7,418 & $32(26-39)$ & $<0.01$ \\
\hline & & Brasfield [6] & $33(30-36)$ & & & \\
\hline & & Bray et al. [19] & $38(36-40)$ & & & \\
\hline & & Lieberman [26] & $30(26-34)$ & & & \\
\hline & & Lieberman [8] & 37 (34-40) & & & \\
\hline & Navy Men & Bray et al. [19] & $46(45-48)$ & 3,341 & & \\
\hline & AF Men & Bray et al. [19] & $47(45-49)$ & 3,193 & & \\
\hline & Marine Men & Bray et al. [19] & $41(39-43)$ & 2,767 & & \\
\hline & \multirow[t]{5}{*}{ Army Women } & Warber et al. [16] & $35(31-39)$ & 1,610 & $40(32-48)$ & $<0.01$ \\
\hline & & Brasfield [6] & $40(31-49)$ & & & \\
\hline & & Bray et al. [19] & $50(47-53)$ & & & \\
\hline & & Lieberman [26] & $28(14-42)$ & & & \\
\hline & & Lieberman et al. 2010 [8] & $41(33-49)$ & & & \\
\hline & Navy Women & Bray et al. [19] & $55(52-58)$ & 1,286 & & \\
\hline & AF Women & Bray et al. [19] & $63(60-66)$ & 1,331 & & \\
\hline & Marine Women & Bray et al. [19] & $53(49-57)$ & 589 & & \\
\hline & Navy SEALS & Schneider et al. [21] & $26(17-35)$ & 1,147 & $37(25-52)$ & $<0.01$ \\
\hline & Army SF & Bovill et al. [12] & $55(46-64)$ & & & \\
\hline & Army Rangers & Lieberman [27] & $23(20-26)$ & & & \\
\hline
\end{tabular}


Table 3 Summary data on prevalence of dietary supplement use by military personnel by gender and service (Continued)

\begin{tabular}{|c|c|c|c|c|c|c|}
\hline \multirow{4}{*}{$\begin{array}{l} \\
\text { Any vitamin } \\
\text { or mineral }\end{array}$} & \multirow[t]{2}{*}{ Army SF } & \multirow{2}{*}{$\begin{array}{c}\text { Lieberman [28] } \\
\text { Lieberman et al. [8] }\end{array}$} & \multicolumn{4}{|l|}{$32(25-39)$} \\
\hline & & & \multicolumn{4}{|l|}{$64(41-87)$} \\
\hline & Army Men & Carlson et al. [14] & $7(1-13)$ & 3,720 & 18(13-26) & $<0.01$ \\
\hline & & Bray et al. [19] & $24(22-26)$ & & & \\
\hline & & Lieberman et al. [8] & $17(15-20)$ & & & \\
\hline & Navy Men & Bray et al. [19] & $27(25-29)$ & 3,341 & & \\
\hline & AF Men & Bray et al. [19] & $25(24-27)$ & 3,193 & & \\
\hline & Marine Men & Bray et al. [19] & $24(22-26)$ & 2,767 & & \\
\hline & Army Women & Bray et al. [19] & $34(31-37)$ & 952 & $29(19-41)$ & 0.01 \\
\hline & & Lieberman et al. [8] & $23(16-30)$ & & & \\
\hline & Navy Women & Bray et al. [19] & $36(33-39)$ & 1,286 & & \\
\hline & AF Women & Bray et al. [19] & $40(37-43)$ & 1,331 & & \\
\hline & Marine Women & Bray et al. [19] & $33(29-37)$ & 589 & & \\
\hline \multirow[t]{6}{*}{ Vitamin C } & Army Men & Brasfield [6] & $17(14-20)$ & 1,609 & $15(12-20)$ & 0.07 \\
\hline & & Lieberman et al. [26] & $13(10-16)$ & & & \\
\hline & Army Women & Brasfield [6] & $15(9-21)$ & 255 & $15(10-21)$ & 0.19 \\
\hline & & Lieberman et al. 2008 [26] & $13(3-23)$ & & & \\
\hline & Army SF & Bovill 2003 [12] & $20(13-27)$ & 271 & 15(8-26) & 0.04 \\
\hline & & Lieberman [28] & $11(6-16)$ & & & \\
\hline \multirow[t]{6}{*}{ Vitamin $\mathrm{E}$} & Army Men & Brasfield [6] & $10(8-12)$ & 514 & $8(5-13)$ & 0.12 \\
\hline & & Lieberman [26] & $6(4-8)$ & & & \\
\hline & Army Women & Brasfield [6] & $7(3-12)$ & 164 & $7(4-12)$ & 0.95 \\
\hline & & Lieberman [26] & $8(1-16)$ & & & \\
\hline & Army SF & Bovill et al. [12] & $12(6-18)$ & 271 & $9(6-15)$ & 0.21 \\
\hline & & Lieberman [28] & $7(3-11)$ & & & \\
\hline \multirow[t]{4}{*}{ Calcium } & Army Men & Brasfield [6] & $12(10-14)$ & 1,194 & $9(4-17)$ & $<0.01$ \\
\hline & & Lieberman [26] & $6(4-8)$ & & & \\
\hline & Army Women & Brasfield [6] & $14(8-20)$ & 164 & $14(10-20)$ & 0.84 \\
\hline & & Lieberman[26] & $15(4-26)$ & & & \\
\hline \multirow[t]{2}{*}{ Iron } & Army Women & Brasfield [6] & $12(6-18)$ & 164 & $12(8-18)$ & 0.72 \\
\hline & & Lieberman [26] & $10(1-19)$ & & & \\
\hline \multirow[t]{2}{*}{ Protein } & Army SF & Bovill et al. [12] & $24(16-32)$ & 271 & $20(13-30)$ & 0.08 \\
\hline & & Lieberman [28] & $16(10-22)$ & & & \\
\hline \multirow{3}{*}{$\begin{array}{l}\text { Protein or } \\
\text { amino acid }\end{array}$} & Navy SEALs & Schneider et al. [21] & $18(15-21)$ & 876 & $21(11-36)$ & $<0.01$ \\
\hline & Army Rangers & Lieberman [27] & $12(5-19)$ & & & \\
\hline & Army SF & Lieberman et al. [8] & $47(23-71)$ & & & \\
\hline \multirow[t]{7}{*}{ Creatine } & Army Men & Brasfield [6] & $16(13-18)$ & 1,194 & $9(3-27)$ & $<0.01$ \\
\hline & & Lieberman [26] & $5(3-7)$ & & & \\
\hline & Navy SEALs & Schneider et al. [21] & $32(22-42)$ & 1,169 & $20(15-25)$ & 0.02 \\
\hline & Army SF & Bovill et al. [12] & $18(11-25)$ & & & \\
\hline & Army Rangers & Deuster et al.[5] & $13(2-24)$ & & & \\
\hline & Army Rangers & Lieberman [27] & $19(16-22)$ & & & \\
\hline & Army SF & Lieberman [28] & $16(10-22)$ & & & \\
\hline
\end{tabular}


Table 3 Summary data on prevalence of dietary supplement use by military personnel by gender and service (Continued)

\begin{tabular}{|c|c|c|c|c|c|c|}
\hline \multirow[t]{9}{*}{ Sport drinks } & \multirow[t]{2}{*}{ Army Men } & Lieberman [26] & $20(16-24)$ & \multirow[t]{2}{*}{1,303} & \multirow[t]{2}{*}{$22(19-25)$} & \multirow[t]{2}{*}{0.22} \\
\hline & & Lieberman et al. [8] & $23(20-26)$ & & & \\
\hline & \multirow[t]{2}{*}{ Army Women } & Lieberman [26] & $28(14-42)$ & \multirow[t]{2}{*}{171} & \multirow[t]{2}{*}{$25(19-32)$} & \multirow[t]{2}{*}{0.24} \\
\hline & & Lieberman et al. [8] & $24(17-31)$ & & & \\
\hline & Navy SEALs & Schneider et al. [21] & $19(11-27)$ & \multirow[t]{5}{*}{1,147} & \multirow[t]{5}{*}{$39(26-55)$} & \multirow[t]{5}{*}{$<0.01$} \\
\hline & Army SF & Bovill et al. [12] & $71(63-79)$ & & & \\
\hline & Army Rangers & Lieberman [27] & $41(38-44)$ & & & \\
\hline & Army SF & Lieberman [28] & $36(28-44)$ & & & \\
\hline & Army SF & Lieberman et al. [8] & $32(10-54)$ & & & \\
\hline \multirow[t]{10}{*}{ Sport bars } & \multirow[t]{3}{*}{ Army Men } & Warber et al. [16] & $8(7-9)$ & \multirow[t]{3}{*}{3,890} & \multirow[t]{3}{*}{$7(5-9)$} & \multirow[t]{3}{*}{0.03} \\
\hline & & Lieberman [26] & $5(3-7)$ & & & \\
\hline & & Lieberman et al. [8] & $6(4-8)$ & & & \\
\hline & \multirow[t]{2}{*}{ Army Women } & Warber et al. [16] & $3(2-5)$ & \multirow[t]{2}{*}{625} & \multirow[t]{2}{*}{$3(2-5)$} & \multirow[t]{2}{*}{0.65} \\
\hline & & Lieberman et al. [8] & $4(1-7)$ & & & \\
\hline & Navy SEALs & Schneider et al. [21] & $40(30-50)$ & \multirow[t]{5}{*}{1,147} & \multirow[t]{5}{*}{ 19(6-46) } & \multirow[t]{5}{*}{$<0.01$} \\
\hline & Army SF & Bovill et al. [12] & $52(43-61)$ & & & \\
\hline & Army Rangers & Lieberman [27] & $6(4-8)$ & & & \\
\hline & Army SF & Lieberman [28] & $15(9-21)$ & & & \\
\hline & Army SF & Lieberman et al. [8] & $7(1-14)$ & & & \\
\hline
\end{tabular}

Abbreviations: $\mathrm{AF}=$ Air Force; SEAL = Sea, Air, Land (US Navy special operations personnel); $\mathrm{SF}=$ Special Forces; $\mathrm{Cl}=$ confidence interval.

that was not included in any other study. Additional data from Bray et al. [19] was included in the table because this investigation was the only one to ask about use of any DS, multivitamins, or any vitamin or mineral supplement $\geq 1$ time/week among Navy, Air Force, and Marine personnel. Meta-analyses indicated that the prevalence of any DS use was relatively consistent among Army studies. Among men in the four military services, use of supplements of any kind ranged from $53 \%$ to $61 \%$ of the surveyed groups; among women, use of supplements of any kind ranged from 66\% to $76 \%$. Female Army officers in training tended to have a much lower use of DSs. Reported supplement use among elite Army and Navy groups was less homogenous ranging from $56 \%$ to $90 \%$ of the surveyed groups, but was, in the main, higher than general military samples.

Table 3 shows prevalence data on MVM use by military personnel. Army studies demonstrated a lack of homogeneity for both men and women. Among men in the four military services, MVM use ranged from $24 \%$ to $47 \%$ with Army men less likely to consume multivitamins than men in the other services. Among women, MVM use ranged from 28\% to $63 \%$ in the four services, again with Army women less likely to consume multivitamins than women in other services. Reported MVM use among elite military groups appeared to be similar to that of Army men but the prevalence values lacked homogeneity and ranged from $26 \%$ to $64 \%$.

Table 3 shows that studies on the use prevalence of any vitamin or mineral supplement by Army men and women lacked homogeneity. Nonetheless, data suggested women used vitamin and mineral supplements more often than men, regardless of service. Among all four services, prevalence of use of any vitamin or mineral supplement among men ranged from $7 \%$ to $27 \%$ while for women this range was $23 \%$ to $40 \%$.

Table 3 shows other DSs that had multiple studies and fit the table criteria (current use or use $\geq 1$ times/week). These included Vitamin C, Vitamin E, calcium, iron, proteins, protein or amino acids, creatine, sport drinks, and sport bars. Multiple studies (i.e., $\geq 2$ ) were limited to Army men, Army women, and some elite military groups. The prevalence of Vitamin C supplementation was 15\% for Army men, Army women, and Special Forces soldiers, although the latter estimate lacked homogeneity. The prevalence of Vitamin E supplementation was similar among Army men and the Special Forces soldiers and slightly higher than that of Army women. Calcium supplementation was slightly more prevalent among Army women compared to Army men, although the two available male studies were not homogeneous. Creatine use appeared more prevalent among samples of elite Army men compared to general male Army samples, although prevalence values differed considerably. When the Navy SEAL study [21] was eliminated from the analysis of creatine prevalence, studies on Rangers and Special Forces soldiers had a $\mathrm{SPE}=19 \%$ and $\mathrm{S} 95 \% \mathrm{CI}=16-21$ ( $\mathrm{p}=0.88$ for homogeneity). Use prevalence of sport drinks was similar among the 
general samples of Army men and women. Meta-analysis suggested that elite military group use of sport drinks was higher than in general Army samples but studies of elite groups lacked homogeneity, with individual study prevalences ranging from $19 \%$ to $71 \%$. The prevalence of sport bar usage was low among samples of Army men and even lower among Army women. Sport bar usage appeared much higher in some samples of elite military groups but there was a lack of homogeneity with prevalences ranging from $6 \%$ to $52 \%$.

The curve fitting procedures did not indicate any significant temporal trends among studies. This can be appreciated by examining the data presented by year in Table 3 .

\section{Discussion}

This review demonstrates that the prevalence of DS use was high among members of the military services. Available data indicated that the self-reported prevalence of DS current use or use $\geq 1$ time/week was slightly lower among Army men (55\%) than among men in the other 3 military services $(\sim 60 \%)$. Female service members reported a higher prevalence of DS use than male service members but again, Army women reported a slightly lower use of DSs of any kind (66\%) compared to women in the other 3 services (71 to 76\%). The pattern of MVM use was similar to that of the overall use prevalence. That is, Army men and women report MVM use prevalences lower than the other services but women in all services report use prevalences higher than their male counterparts in the same service. Prevalence estimates varied in groups of elite service members (Army Rangers, Army Special Forces, and Navy SEALs) but generally, the use prevalence of supplements of any kind was higher (56\% to $90 \%$ ) than that of other service members. Interestingly, male and female Army officers in training tended to have a lower prevalence of DS use, possibly related to the fact that they were eating in military dining facilities and had busy training schedules.

MVM appeared to be the most commonly used DS among the general population of military personnel with $24 \%$ to $47 \%$ of male and $28 \%$ to $63 \%$ of female service members using these supplements. This is in consonance with data on athletes $[49,50]$ and the general US population [51-56] where MVM are also the most commonly consumed DS. Table 4 shows summary data from the National Health Interview Surveys (NHIS) [54,55,57] and the National Health and Nutrition Surveys (NHANES) [58-61], both of which are nationally representative samples of the general US population. It was difficult to directly compare military MVM prevalence data to that of these national surveys because the military and civilian studies differed in terms of the reporting timeframe, methods used to collect the data, and the years when the data were collected. Despite these issues, it was possible to make some general observations. The NHANES and
NHIS surveys indicated that women were more likely to use DSs than men, in agreement with the military data. Both NHANES and NHIS surveys observed a temporal trend indicating that DS use increased over time, although the most recent NHANES data suggested a leveling off $[60,61]$. No such temporal trends could be discerned in the military data, possibly because of the shorter time over which the studies were conducted and lack of questionnaire standardization across studies. Use of MVM appeared to be higher in the military compared to these civilian samples.

Among military personnel, the most common rationale for taking MVMs was to promote general health with 76\% of individuals reporting this reason in one study [8]. Most MVM supplements contain at least 10 vitamins and/or minerals with a great range of dosages [62]. Systematic and narrative reviews on the effects of MVM supplements on health have indicated that there is little convincing evidence that these DSs influence the incidence of cataracts, cardiovascular disease, diabetes [63-65], or cancers of the prostate, lungs, or breast [64,66-68]. On the other hand some studies suggest MVM supplements may improve cognitive functioning $[69,70]$, reduce infection risk in older individuals [71], reduce colon cancer risk [72] and, when used for primary prevention, reduce the risk of all-cause mortality [73], although results are not consistent [65]. The determination of the safety of vitamins and minerals differs from that of other substances like toxins or other chemicals because a certain level of vitamins and minerals are needed for good health but above or below that level, an adverse effect may occur. Thus, there is a "U-shaped" relationship between the dosage and the likelihood of adverse effects and dose-response curve will differ for different DSs [74].

Use prevalence of individual vitamins and minerals were less often reported in military studies but a few studies of Army personnel had examined Vitamin C, Vitamin E, calcium, and iron [6,12,26]. Generally, the use prevalence of Vitamin $C$ was similar among Army men, women, and Special Forces Soldiers (15\%). Vitamin E use prevalence was about the same among Army men, Army women, and elite groups (7-12\%). Women appeared more likely to supplement with calcium (14\%) compared to Army men (9\%). Table 4 provides data on Vitamin C, Vitamin E, and calcium supplementation from the NHIS. Again, comparisons with the military data are limited for reasons cited above. Nonetheless, the NHIS and NHANES general trends were similar to the military data in that men and women were about equally likely to supplement with Vitamins $\mathrm{C}$ and $\mathrm{E}$ and women were more likely to use calcium.

Vitamin $\mathrm{C}$ and $\mathrm{E}$ are antioxidant vitamins and they have been studied in disease states like cancer, cardiovascular disease, and macular degeneration where oxidative stress mechanisms produce cellular damage $[75,76]$. 
Table 4 Summary data on DS Use in United States national surveys

\begin{tabular}{|c|c|c|c|c|c|c|c|c|c|c|c|c|c|c|}
\hline \multirow[t]{3}{*}{ Survey } & \multirow[t]{3}{*}{ Study } & \multirow{3}{*}{$\begin{array}{l}\text { Survey } \\
\text { year(s) }\end{array}$} & \multirow[t]{3}{*}{$\mathrm{N}$} & \multirow{3}{*}{$\begin{array}{l}\text { Reporting } \\
\text { timeframe }\end{array}$} & \multicolumn{10}{|c|}{ Prevalence (\%) } \\
\hline & & & & & \multicolumn{2}{|c|}{ Any VM } & \multicolumn{2}{|c|}{ Multivitamin } & \multicolumn{2}{|c|}{ Vitamin C } & \multicolumn{2}{|c|}{ Vitamin E } & \multicolumn{2}{|c|}{ Calcium } \\
\hline & & & & & Men & Women & Men & Women & Men & Women & Men & Women & Men & Women \\
\hline \multirow{3}{*}{$\begin{array}{l}\text { National Health Interview } \\
\text { Survey (NHIS) }\end{array}$} & Subar \& Block [57] & 1987 & 9,160 ठૈ, 12,920 우 & Daily Use & 19 & 27 & 15 & 20 & 7 & 8 & 4 & 5 & 3 & 10 \\
\hline & Slesinski et al. [54] & 1992 & 5,120 ふ, 6,885 ㅇ & Daily Use & 20 & 27 & 17 & 22 & 7 & 8 & 4 & 5 & 2 & 8 \\
\hline & Millen et al. [55] & 2000 & 34,085 ठ \& $q$ & Daily Use & 29 & 39 & 24 & 33 & 10 & 12 & 10 & 13 & 4 & 17 \\
\hline \multirow{4}{*}{$\begin{array}{l}\text { National Health and Nutrition } \\
\text { Survey (NHANES) }\end{array}$} & Koplan et al. [58] & 1976-1980 & 5,915 ふ̊, 6,588우 & Use $\geq 1$ time/wk & 30 & 40 & ND & ND & ND & ND & ND & ND & ND & ND \\
\hline & Balluz et al. [59] & 1988-1994 & $33,905 \precsim \&$ \& & Use in Last Month & 35 & 44 & ND & ND & ND & ND & ND & ND & ND & ND \\
\hline & Radimer et al. [60] & $1999-2000$ & 2,260 ô, 2,602 q & Use in Last month & 46 & 57 & 32 & 38 & 12 & 13 & 12 & 14 & 4 & 16 \\
\hline & Kennedy et al. [61] & $2007-2008$ & 3,364 శै \& $q$ & Use in Last Month & 42 & 54 & ND & ND & ND & ND & ND & ND & ND & ND \\
\hline
\end{tabular}

Abbreviations: $\mathrm{VM}=$ vitamin and/or mineral; $\hat{\gamma}=$ men; $\uparrow=$ women; $\mathrm{ND}=$ no data; $\mathrm{wk}=$ week. 
In systematic reviews, Vitamin $\mathrm{C}$ and $\mathrm{E}$ supplementation either alone or in combination with other substances did not reduce the incidence of cardiovascular disease $[64,75,77]$ or prostate cancer [66]. Supplemental Vitamin C may have modestly reduced the risk of breast cancer but Vitamin $\mathrm{E}$ did not [78]. Vitamin C and E from any source (diet or supplements) appeared to reduce the incidence of endometrial cancer [79]. Calcium supplementation with Vitamin D reduced stress fracture risk in basic training [80].

The prevalence of herbal supplement use by military personnel was small, usually $5 \%$ or less of the groups surveyed $[6,17,23,29,33,39,45]$, although ginseng use was $14 \%$ in one Army study [6]. The nationally representative NHIS data from 2007 indicated that 17\%, 12\%, 12\%, and $11 \%$ of the surveyed group had used Echinacea, garlic supplements, ginseng, and ginkgo biloba, respectively, in the last 30 days [81]. In athletic groups, $2 \%$ to $10 \%$ reported current use of ginseng and 4 to $7 \%$ reported current use of Echinacea [50,82,83].

Sport drinks are a general category of beverages that contain water, carbohydrates, minerals, and electrolytes, and may contain small amounts of vitamins. They are designed to be used during and after sport and exercise activities for rehydration, replacement of electrolytes lost during sweating, and energy (i.e., supply carbohydrates during activity or replenish muscle glycogen post-activity) $[84,85]$. Sport drinks appeared to be a very common nutritional supplement used by elite military groups, although the use prevalence range was wide (19\% to $71 \%$ ) in the various investigations. Sport drink use prevalence was also high among the general male and female military population $(\sim 23 \%)$ but this did not exceed the use prevalence for MVMs in these groups. The relatively high use of sport drinks among elite military groups is in consonance with studies of elite athletes showing that the use of sport drinks exceeds that of multivitamins [86-88]. Data from the NHIS indicated that $22 \%$ reported the use of sport or energy drinks $\geq 1$ time/week but that survey did not provide data on the two beverages separately [89]. Data from NHANES indicated an almost tripling in the use of sport and energy drinks (combined) among adolescents and young adults (12 to 34 years of age) over the 1999 to 2008 period [90].

Creatine was a DS with relatively high use prevalence among elite service members (20\%) and among Army men (14\%), although the two studies on Army men had widely varying prevalence values $[6,26]$. The 2007 NHIS survey reported a $3 \%$ use prevalence in the general US population [81]. Studies on athletes have found that creatine use prevalence is highly variable and dependent on the sport. Athletes in strength and power sports (e.g., weightlifting, football, track and field) used creatine to a greater extent than those involved in endurance activities [91-94]. Research has generally shown that creatine supplementation can improve strength and performance in short-term, high intensity physical activities [95-98]. In combination with resistance training, creatine increased maximal muscle strength to a greater extent than resistance training alone [99].

In elite military groups, the use prevalence for protein supplements was about $20 \%$ but varied widely, between $12 \%$ and $47 \%[8,12,21,27]$. The only study to report on the protein supplementation in the general male army population found a use prevalence of 13\% [26], while another study reporting on combined proteins and amino acids found a use prevalence of $20 \%$ [8]. One national survey (Health and Diet Survey conducted by the US Food and Drug Administration) reported that about 1\% of the total sample had used amino acid supplements in the last year [56]. The Recommended Daily Allowance for protein is 0.8 gm $\bullet \mathrm{kg}$ body weight ${ }^{-1} \bullet^{-1 a y}{ }^{-1}$ [100]. However, summaries of studies indicated that the daily average intake of protein among strength-trained athletes was $2.1 \mathrm{gm} \mathrm{kg}^{-1} \bullet$ day $^{-1}$ [101] while that among endurance athletes was $1.8 \pm 0.4 \mathrm{gm} \mathrm{kg}^{-1} \bullet \mathrm{day}^{-1}$ for men and $1.2 \pm$ $0.03 \mathrm{gm} \bullet \mathrm{kg}^{-1} \bullet \mathrm{day}^{-1}$ for women [102]. A recent consensus statement on the efficacy of protein supplementation in military personnel recommended 1.5 to $2.0{\text { gm } \bullet \mathrm{kg}^{-1} \bullet \text { day }}^{-1}$ for service members involved in substantially increased metabolic demand and 1.2 to 1.5 gm $\bullet \mathrm{kg}^{-1} \bullet$ day ${ }^{-1}$ for older service members [103]. A meta-analysis of 22 studies suggested that protein supplementation $\left(>1.2{\text { gm } \bullet \mathrm{kg}^{-1} \bullet \text { day }}^{-1}\right)$ with resistance training resulted in modestly greater gains in fat-free mass $(0.7 \mathrm{~kg}, 95 \% \mathrm{CI}=0.5-0.9 \mathrm{~kg})$ and strength when compared to training without protein supplementation [104].

Three studies reported on energy drink consumption among soldiers [42] and among soldiers and marines deployed in Afghanistan $[9,44]$. Prevalence of energy drink usage was $25 \%$ among female soldiers [42] and $41 \%$ to $45 \%$ among male soldiers and marines $[9,42,44]$. There are about 500 brands of energy drinks available worldwide and about 200 are available in the US $[105,106]$. Literature reviews examining commonly available energy drinks found that virtually all contained caffeine, taurine, and BVitamins, while other common ingredients contained in most included guarana, ginseng, sugars, and carnitine. Other ingredients found in some energy drinks include ginkgo biloba, milk thistle, branched-chain amino acids, choline, chromium, green tea, hornet saliva, inositol, yerba mate, triglycerides, proline, pyruvate, royal jelly, schizandra, aloe vera, bee pollen, borage oil, and stabilized oxygen. Reviews have generally concluded that except for some relatively weak evidence for glucose and guarana, there was little evidence for a positive effect on cognitive or physical performance for any of those ingredients other than caffeine [106-108]. Caffeine has been shown to increase performance during long-term exercise, shorter- 
term high intensity exercise (60-180 sec), and high intensity intermittent exercise, but effects on muscle strength are equivocal. Caffeine may produce ergogenic effects through a variety of mechanisms that include increasing fat oxidation (thus sparing glucose and muscle glycogen during long-term exercise), central nervous system stimulation, a direct action on muscles, and/or a competitive inhibition with adenosine. The adenosine mechanism involves caffeine occupying adenosine sites in the central nervous system which increase catecholamine release and lipolysis. Tolerance to caffeine has often been reported and may be associated with the up-regulation of adenosine receptors [109-112].

Of interest were the studies on Marine Corps and Air Force trainees that asked about lifetime prevalence of DS use [36,39]. About $82 \%$ of the participants in these two studies were men. The lifetime prevalence of any DS use was $41 \%$ [36] and 50\% [39]. These prevalences were lower than those reported by Bray et al. [19] for longer-serving Marine and AF personnel ( 60\%). These data suggest that a number of service members have used DSs before entering the Air Force or Marine Corps but that prevalence becomes higher once individuals spend time in these services. Military service may increase DS use because of the demands of the occupations and the belief that DSs will improve health and increase performance on occupational tasks.

In the nine investigations that examined the prevalence of DS use in elite military units [5,8,12,21,22,27,28,38,45], all but two $[22,38]$ reported a higher use of DSs (any use) among these elite service members compared to non-elite male military samples. This is similar to results found for elite athletes where athletes participating at higher levels of competition (Olympic, national, or international level) were more likely to use DSs than those competing as recreational athletes [49]. Elite athletes and elite service members may be similar in that they seek to gain additional physical advantages from the use of DSs.

Five studies provided information on the reasons that service members used DSs $[6,8,9,19,33]$. In four of the five, "general health" was listed as the reason with the highest frequency with performance enhancement listed as second most common (first in Cassler et al. study [9] of deployed marines). Thus, service members reported using DSs for the same reason as civilians, $[10,11]$ but a second very common reason was performance enhancement which is seldom mentioned in civilian investigations. In this sense, service members are like athletes who also report performance enhancement as a high frequency reason for DS use [113-115]. Like athletes, service members' occupational tasks require a high level of physical performance; they are unlike most athletes in that their activity may be performed in hostile locations and under adverse and austere environmental conditions.
Future studies on the prevalence of DS use should consider five major issues. First, the definition of DSs should be clearly stated on the questionnaire instructions. The legal definition provided by The Dietary Supplement Health and Education Act of 1994 [2] can serve as a standard. Second, studies should be specific about the types of DSs used by study participants. Reporting in general categories like "antioxidant", "energy", "herbal", "bodybuilding", and the like does not provide the specificity needed for comparisons across studies and the identification of specific DS use. Third, the reporting timeframe should be specific and include several periods. The most useful reporting timeframes appear to be daily, 2-6 times/week, 1 time/ week and 1 time/month. Fourth, the response rate of survey should be specified and, if possible, characteristics of respondents and non-respondents should be described so that possible bias can be assessed. Finally, studies are needed that use the same experimental methods to compare DS use across all the military services over time.

There were limitations to this review. Studies differed on the reporting timeframe, questionnaire construction, and supplement definitions which made it difficult to directly compare results across all studies. In the metaanalysis, an attempt was made to control for the reporting timeframe by only examining studies asking service members about current supplement use or use $\geq 1$ day/week. We only examined specific DSs and did not include DSs that were included in broad categories (e.g., antioxidant, ergogenics, bodybuilding). Thus, we may have underestimated DSs in some categories, although most unique studies $(\mathrm{n}=24,72 \%)$ did report use of "any" DS. Some questionnaires involved "checklists" of specific DSs that may have elicited better subject recall than open-ended questions asking subjects to list the DSs that they used. In most studies, the actual questionnaire structure and/or questionnaire items were not specified and the only apparent fact was that the questionnaire did or did not focus on DSs. It is possible that some service members may have been involved in one or more surveys but the number of these individuals would likely have been very small. Some values had to be estimated from graphic presentations which could have resulted in small errors. The analyses of temporal trends depended on the publication year which was likely not the year that the data were collected. Other problems common to self-reporting included the accuracy of subject recall and the possible reluctance of some individuals to report specific DSs that they used.

\section{Conclusion}

In conclusion, this review provided a comprehensive overview of military DS use by gender and type of military service. It demonstrated that Army personnel tended to use DSs and MVM less than other service members but that regardless of service, the use of any DS and MVMs are 
higher among women than men. Military personnel's use of herbal supplements is small, $<5 \%$ in most investigations. Elite military men appeared to use DSs and sport drinks more than other service members.

\section{Abbreviations}

US: United States; DS: Dietary supplement; MVM: Multivitamin/multimineral; SPE: Summary prevalence estimate; S95\% Cl: Summary 95\% confidence Interval; SEAL: Sea, air, land (Navy Special Operations Personnel); DTIC: Defense technical information center; NHANES: National Health and Nutrition Survey; NHIS: National Health Interview Survey; 95\% Cl: 95\% confidence interval.

\section{Competing interests}

The authors have no competing interests.

\section{Authors' contributions}

JJK obtained references, compiled the data, performed the methodology quality review, performed the statistical analysis, interpreted the data, and drafted the manuscript. RS obtained references, compiled the data, performed the methodology quality review, assisted with the statistical analysis and data interpretation, and helped draft the manuscript. SSH performed the methodology quality review, assisted in data interpretation, and helped draft the manuscript. KA, EF, and HRL assisted with data interpretation and helped draft the manuscript. All authors read, commented on, and approved the final manuscript.

\section{Authors' information}

The views, opinions, and/or findings contained in this report are those of the authors and should not be construed as official Department of the Army position, policy, or decision, unless so designated by other official documentation. Approved for public release; distribution is unlimited.

\section{Acknowledgements}

We would like to thank Claudia Coleman and Dr Wayne Askew who assisted us in obtaining references. This research was supported in part by an appointment to the Knowledge Preservation Program at the US Army Research Institute of Environmental Medicine (USARIEM) and the Army Institute of Public Health (AIPH) administered by the Oak Ridge Institute for Science and Education through an interagency agreement between the U.S. Department of Energy and USARIEM. Funding was also provided by the Department of Defense Center for Dietary Supplement Research and the Medical Research and Development Command.

\section{Author details}

'US Army Research Institute of Environmental Medicine, Natick, MA, USA. ${ }^{2}$ US Army Institute of Public Health, Aberdeen Proving Ground, MD, USA. ${ }^{3}$ Serenity Hill Nutrition, Street, MD, USA. ${ }^{4}$ Oak Ridge Institute for Science and Education, Belcamp, MD, USA. ${ }^{5}$ Research Physiologist, ORISE Knowledge Preservation Fellow, USARIEM, 42 Kansas Street, Natick, MA, USA.

\section{Received: 23 October 2013 Accepted: 25 April 2014}

\section{Published: 2 May 2014}

\section{References}

1. Strengthening knowledge and understanding of dietary supplements. [http://ods.od.nih.gov/About/DSHEA_Wording.aspx], accessed 4 February 2013.

2. Dietary Supplement Health and Education Act of 1994. [http://wnw.fda.gov/ RegulatoryInformation/Legislation/FederalFoodDrugandCosmeticActFDCAct/ SignificantAmendmentstotheFDCAct/ucm148003.htm], accessed 11 March 2013.

3. Saldanha LG: The dietary supplement marketplace. Constantly evolving. Nutr Today 2007, 42(2):52-54.

4. Considering a post-DSHEA World. Nutr Bus J 2012, 17(5/6):1, 3-9.

5. Deuster PA, Sridhar A, Becker WJ, Coll R, O'Brien KK, Bathalon G: Health assessment of U.S. Army Rangers. Mil Med 2003, 168(1):57-62

6. Brasfield K: Dietary supplement intake in the active duty enlisted population. US Army Med Dep J 2004, 44-56.

7. Boos C, Wheble GAC, Campbell MJ, Tabner KC, Woods DR: Self-administration of exercise and dietary supplements in deployed British military personnel during operation TELIC 13. J R Army Med Corps 2010, 156(1):32-36.
8. Lieberman HR, Stavinoha TB, McGraw SM, White A, Hadden LS, Marriott BP: Use of dietary supplements among active-duty US Army soldiers. Am 」 Clin Nutr 2010, 92(4):985-995

9. Cassler NM, Sams R, Cripe PA, McGlynn AF, Perry AB, Banks BA: Patterns and perceptions of supplement use by U.S. Marines deployed to Afghanistan. Mil Med 2013, 178(6):659-664.

10. Bailey RL, Gahche JJ, Miller PE, Thomas PR, Dwyer JT: Why US adults use dietary supplements. JAMA Int Med 2013, 173(3):355-361.

11. Dickinson A, Bonci L, Boyon N, Franco JC: Dietitians use and recommend dietary supplements: report of a survey. Nutr J 2012, 11:14.

12. Bovill ME, Tharion WJ, Lieberman HR: Nutrition knowledge and supplement use among elite U.S. Army soldiers. Mil Med 2003, 168(12):997-1000.

13. Loney PL, Chambers LW, Bennett KI, Roberts JG, Stratford PW: Critical appraisal of health research literature: prevalence or incidence of a health problem. Chronic Dis Can 2000, 19(4):170-176.

14. Carlson DE, Dugan T, Buchbinder J, Allegetto J, Schnakenberg DD: Nutritional Assessment of the Ft Riley Non-Commissioned Officer Academy Dining Facility. Natick MA: US Army Research Institute of Environmental Medicine Technical Report No. T14-87; 1987.

15. Klicka MV, Sherman DE, King N, Friedl KE, Askew EW: Nutritional Assessment of Cadets at the U.S. Military Academy. Part 2. Assessment of Nutritional Intake. Natick MA: US Army Research Institute of Environmental Medicine Technical Report No. T94-1; 1994

16. Warber J, McGraw S, Kramer FM, Lesher L, Johnson W, Cline A: The Army Food and Nutrition Survey. Natick MA: US Army Research Institute of Environmental Medicine Technical Report No. XX-99; 1999.

17. Shanks K: Prevalence of Herbal Therapy use in Active Duty Air Force Women. Bethesda MD: Uniformed Services University of the Health Sciences Technical Report No. C101-88; 2001.

18. Castillo EM, Hurtado SL, Shaffer RA, Rock CL, Brodine SK: Dietary Supplement use in a Physically Active Population. San Diego CA: Naval Health Research Center Technical Report; 2004.

19. Bray RM, Hourani LL, Olmsted KLR, Witt M, Brown JM, Pemberton MR, Marsden ME, Marriott B, Scheffler S, Vandermass-Peeler R, Weimer B, Calvin S, Bradshaw M, Close K, Hayden D: 2005 Department of Defense Survey of Health Related Behaviors Among Active Duty Personnel Research Triangle. Park NC: Research Triangle Institute Technical Report No. RT//7841/106-FR; 2006.

20. Wells TS, Webb TS: Modifiable Characteristics Associated With the Training Success Among US Air Force Tactical Control Party Candidates. Wright-Patterson Air Force Base OH: Air Force Research Laboratory Technical Report; 2010.

21. Schneider K, Hervig L, Ensign WY, Prusaczyk WK, Goforth HW: Use of supplements by U.S. Navy seals. Med Sci Sports Exerc 1998, 30(5):60.

22. McGraw SM, Therion WJ, Lieberman HR: Use of nutritional supplements by U.S. Army Rangers. FASEB J 2000, 14(4):A742.

23. Corum S: Findings of Recent Surveys on Dietary Supplements Use by Military Personnel and the General Population (Appendix C). In Use of Dietary Supplements by Military Personnel. Edited by Greenwood MRC, Oria M. Washington DC: National Academy Press; 2008:384-385.

24. French A: Findings of Recent Surveys on Dietary Supplements Use by Military Personnel and the General Population (Appendix C). In Use of Dietary Supplements by Military Personnel. Edited by Greenwood MRC, Oria M. Washington DC: National Academy Press; 2008:386-387.

25. Marroitt BM: Findings of Recent Surveys on Dietary Supplements Use by Military Personnel and the General Population (Appendix C). In Use of Dietary Supplements by Military Personnel. Edited by Greenwood MRC, Oria M. Washington DC: National Academy Press; 2008:404-405.

26. Lieberman H: Findings of Recent Surveys on Dietary Supplements Use by Military Personnel and the General Population (Appendix C). In Use of Dietary Supplements by Military Personnel. Edited by Greenwood MRC, Oria M. Washington DC: National Academy Press; 2008:398-399.

27. Lieberman H: Findings of Recent Surveys on Dietary Supplements Use by Military Personnel and the General Population (Appendix C) Army Rangers. In Use of Dietary Supplements by Military Personnel. Edited by Greenwood MRC, Oria M. Washington DC: National Academy Press; 2008:400-401.

28. Lieberman H: Findings of Recent Surveys on Dietary Supplements Use by Military Personnel and the General Population (Appendix C), Special Forces. In Use of Dietary Supplements by Military Personne. Edited by Greenwood MRC, Oria M. Washington DC: National Academy Press; 2008:400-401.

29. Lieberman H: Findings of Recent Surveys on Dietary Supplements Use by Military Personnel and the General Population (Appendix C), Army War 
College. In Use of Dietary Supplements by Military Personnel. Edited by Greenwood MRC, Oria M. Washington DC: National Academy Press; 2008:402-403.

30. Johnson AE: Findings of Recent Surveys on Dietary Supplements Use by Military Personnel and the General Population (Appendix C). In Use of Dietary Supplements by Military Personnel. Edited by Greenwood MRC, Oria M. Washington DC: National Academy Press; 2008:414-415.

31. Thomasos CJ: Findings of Recent Surveys on Dietary Supplements Use by Military Personnel and the General Population (Appendix C). In Use of Dietary Supplements by Military Personnel. Edited by Greenwood MRC, Oria M. Washington DC: National Academy Press; 2008:406-407.

32. Klicka MV, King N, Lavin PT, Askew EW: Assessment of dietary intake of cadets at the US Military Academy at West Point. J Am Coll Nutr 1996, 15(3):273-282.

33. Kennedy J, Arsenault J: Dietary supplement use in U.S. Army Special Forces Operations Candidates. Mil Med 1999, 164(7):495-501.

34. Sheppard HL, Raichada SM, Kouri KM, Stenson-Bar-Maor L, Branch JD: Use of creatine and other supplements by members of civilian and military health clubs: a cross-sectional survey. Int J Sport Nutr Exerc Metab 2000, 10:245-259.

35. Arsenault JE, Cline AD: Nutrition intakes and characteristics of normal weight, female personnel consuming foods reduced in fat or energy content. Appetite 2000, 34:227-233.

36. Stevens $M B$, Olsen $C$ : Ergogenic supplements and health risk behaviors. J Fam Pract 2001, 50(8):696-699.

37. Smith TC, Ryan MAK, Smith D, Reed RJ, Riddle JR, Gumbs GR, Gray GC: Complementary and alternative medicine use among US Navy and Marine Corps personnel. BMC Complement Altern Med 2007, 7:16.

38. Johnson AE, Haley CA, Ward JA: Hazards of dietary supplement use. J Spec Oper Med 2007, 7(1):30-38.

39. Young CR, Stevens MB: Sports and nutritional supplement use in USMC recruits: a pilot study. Mil Med 2009, 174(2):158-161

40. Boos CJ, Simms P, Morris FR, Fertout M: The use of exercise and dietary supplements among British soldiers in Afghanistan. J R Army Med Corps 2011, 157(3):229-232.

41. Carvey CE, Farina EK, Lieberman HR: Confidence in the efficacy and safety of dietary supplements among United States active duty Army personnel. BMC Complement Altern Med 2012, 12:182.

42. Lieberman HR, Stavinoha T, McGraw S, White A, Hadden L, Marriott BP. Caffeine use among active duty US Army soldiers. J Acad Nutr Diet 2012, 112(6):902-912.

43. Jacobson IG, Horton JL, Smith B, Wells TS, Boyko EJ, Lieberman HR, Ryan MAK, Smith TC: Bodybuilding, energy, and weight loss supplements are associated with deployment and physical activity in U.S. military personnel. Ann Epidemiol 2012, 22(5):318-330.

44. Toblin RL, Clarke-Walper K, Kok BC, Sipos ML, Thomas JL: Energy drink consumption and its association with sleep problems among U.S. service members on a combat deployment-Afghanistan. MMWR 2012, 61(44):895-898.

45. Kjertakov M, Hristovski R, Racaj M: The use of dietary supplement among soldiers from the Macedonian Special Operations Regiment. J Spec Oper Med 2013, 13(1):19-24.

46. Hill TM, Nelson RA, Consolazio CF, Canham JE: Nutrient Intake of the Repatriated United States Army, Navy and Marine Corps Prisoners-of-war of the Vietnam War. Presidio of San Francisco: Letterman Army Institute of Research Technical Report No. 61; 1978.

47. Jaghab S: Findings of Recent Surveys on Dietary Supplements Use by Military Personnel and the General Population (Appendix C). In Use of Dietary Supplements by Military Personnel. Edited by Greenwood MRC, Oria M. Washington DC: National Academy Press; 2008:394-395.

48. Institutes of Medicine: Use of Dietary Supplements by Military Personnel. Washington DC: Institute of Medicine; 2008.

49. Sobal J, Marquart LF: Vitamin/mineral supplement use among athletes: a review of the literature. Int J Sports Nutr 1994, 4:320-334.

50. Huang SH, Johnson K, Pipe AL: The use of dietary supplements and medications by Canadian athletes at the Atlanta and Sydney Olympic Games. Clin J Sport Med 2006, 16(1):27-33.

51. Rhee KS, Stubbs AC: Health food users in two Texas cities. J Am Diet Assoc 1976, 68:542-545.

52. Schutz HG, Read M, Bendel R, Bhalla VS, Harrill I, Monagle JE, Sheehan ET, Standal BR: Food supplement usage in seven Western states. Am J Clin Nutr 1982, 36:897-901.
53. Block G, Cox C, Madans J, Schreiber GB, Melia N: Vitamin supplement use, by demographic characteristics. Am J Epidemiol 1988, 127(4):297-309.

54. Slesinski MJ, Subar AF, Kahle LL: Trends in the use of vitamin and mineral supplements in the United States: the 1987 and 1992 National Health Interview Surveys. J Am Diet Assoc 1995, 95(8):921-923.

55. Millen AE, Dodd KW, Subar AF: Use of vitamin, mineral nonvitamin and nonmineral supplements in the United States: the, 1992 and 2000 National Health Interview Survey results. J Am Diet Assoc 1987, 2004(104):942-950.

56. Timbo BB, Ross MP, McCarthy PV, Lin CTJ: Dietary supplements in a national survey: prevalence of use and reports of adverse events. J Am Diet Assoc 2006, 106:1966-1974.

57. Subar AF, Block G: Use of vitamin and mineral supplements: demographics and amount of nutrients consumed. Am J Epidemiol 1990, 132(6):1091-1101.

58. Koplan JP, Annest JL, Layde PM, Rubin GL: Nutrient intake and supplementation in the United States (NHANES II). Am J Public Health 1986, 76(3):287-289.

59. Balluz LS, Kieszak SM, Philen RM, Mulinare J: Vitamin and mineral supplement use in the United States. Arch Fam Med 2000, 9:258-262.

60. Radimer K, Bindewald B, Hughes J, Ervin B, Swanson C, Picciano MF: Dietary supplement use by US adults: data from the National Health and Nutrition Examination Survey,1999-2000. Am J Epidemiol 2004, 160(4):339-349.

61. Kennedy ET, Luo H, Houser RF: Dietary supplement use pattern of US adult population in the,2007-2008 National Health and Nutrition Survey (NHANES). Ecol Food Nutr 2013, 52(76):84.

62. Huang HY, Caballero B, Chang S, Alberg AJ, Semba RD, Schneyer C, Wilson RF, Cheng TY, Prokopowicz G, Barnes GJ, Vassy J, Bass EB: Multivitamin/Multimineral Supplements and Prevention of Chronic Diseases. Rockville MD: Agency for Healthcare Research and Quality (US) Technical Report No. 139; 2007.

63. Huang HY, Caballero B, Chang S, Alberg AJ, Semba RD, Schneyer C, Wilson RF, Cheng TY, Vassy J, Prokopowicz G, Barnes GJ, Bass EB: The efficacy and safety of multivitamin and mineral supplement use to prevent cancer and chronic disease in adults: a systematic review for a National Institute of Health State-of-the-Science Conference. Ann Int Med 2006, 145:372-385.

64. Fortmann SP, Burda BU, Senger CA, Linn JS, Whitlock EP: Vitamin and mineral supplements in the primary prevention of cardiovascular disease and cancer: an updated systematic evidence review for the US Preventive Services Task Force. Ann Int Med 2013, 159(12):824-834.

65. Sesso HD, Christen WG, Bubes V, Smith JP, MacFadyen J, Schvartz M, Manson JE, Glynn RJ, Buring JE, Gaziano JM: Multivitamins in the prevention of cardiovascular disease in men. The Physicians' Health Study II Randomized Controlled Trial. JAMA 2012, 308(17):1751-1760.

66. Stratton J, Godwin M: The effect of supplemental vitamins and minerals on the development of prostate cancer: a systematic review and meta-analysis. Fam Pract 2011, 28:243-252.

67. Slatore CG, Littman AJ, Au DH, Satia JA, White E: Long-term use of supplemental multivitamins, Vitamin C, Vitamin E, and folate does not reduce the risk of lung cancer. Am J Respir Crit Care Med 2007, 177:524-530.

68. Cho E, Hunter DJ, Spiegelman D, Albanes D, Beeson WL, VandenBrandt PA, Colditz GA, Feskanich D, Folsom AR, Fraser GE, Freudenheim JL, Giovannucci E, Goldbohm RA, Graham S, Miller AB, Rohan TE, Sellers TA, Virtamo J, Willett WC, Smith-Warner SA: Intakes of Vitamins A, C, and E and folate and multivitamins and lung cancer: a pooled analysis of 8 prospective studies. Int J Cancer 2006, 118:970-978.

69. Kennedy DO, Haskell CF: Vitamins and cognition. What is the evidence? Drugs 2011, 71(15):1957-1971.

70. Grima NA, Pase MP, MacPherson H, Pipingas A: The effects of multivitamins on cognitive performance: a systematic review and meta-analysis. J Alzheimer's Dis 2012, 29:561-569.

71. Stephens Al, Avenell A: A systematic review of multivitamin and multimineral supplementation for infection. J Hum Nutr Dietet 2006, 19:179-190.

72. Park Y, Spiegelman D, Hunter DJ, Albanes D, Bergkvist L, Buring JE, Freudenheim JL, Goldbohm E, Hernack L, Kato I, Krogh V, Leitzmann MF, Limburg PJ, Marshall JR, McCullough ML, Miller AB, Rohan TE, Schatzkin A, Shore R, Sieri S, Stampfer MJ, Virtamo J, Weijenberg M, Weijenberg WC, Willett WC, Wolk A, Zhang SM, Smith-Warner SA: Intakes of vitamins A, C and $E$ and use of multivitamin supplements and risk of colon cancer: a pooled analysis of prospective cohort studies. Cancer Causes Control 2010, 21(11):1745-1757. 
73. MacPherson H, Pipingas A, Pase MP: Multivitamin-multimineral supplementation and mortality: a meta-analysis of randomized controlled trials. Am J Clin Nutr 2013, 97:437-444.

74. Mulholland CA, Benford DJ: What is known about the safety of multivitamin-multimineral supplements for the generally healthy population? Theoretical basis for harm. Am J Clin Nutr 2007, 85(Suppl):318S-322S

75. Nunez-Cordoba JM, Martinez-Gonzales MA: Antioxidant vitamins and cardiovascular disease. Curr Top Med Chem 2011, 11:1861-1869.

76. Evans JR, Lawrenson JG: Antioxidant vitamin and mineral supplements for slowing the progression of age-related macular degeneration (review). Cochrane Database Syst Rev 2012, 11:CD000254. doi:10.1002/14651858. CD000254.pub3.

77. Riccioni G, D'Orazio N, Salvatore C, Franceschelli S, Pesce M, Speranza L: Carotenoids and Vitamins $\mathrm{C}$ and $\mathrm{E}$ in the prevention of cardiovascular disease. Int J Vit Nutr Res 2012, 82(1):15-26.

78. Fulan H, Changxing J, Baina WX, Wencui Z, Chunging L, Fan W, Sandan L, Dianjun S, Tong W, Da P, Yashuang Z: Retinol, Vitamins A, C, and E and breast cancer risk: a meta-analysis and meta-regression. Cancer Causes Control 2011, 22:1383-1396.

79. Bandera EV, Gifkins DM, Moore DF, McCullough ML, Kushi LH: Antioxidant vitamins and the risk of endrometrial cancer: a dose-response metaanalysis. Cancer Causes Control 2009, 20(5):699-711.

80. Lappe J, Cullen D, Haynatzki G, Recker R, Ahlf R, Thompson K: Calcium and Vitamin D supplementation decrease incidence of stress fractures in female Navy recruits. J Bone Miner Res 2008, 23:741-749.

81. Wu CH, Wang CC, Kennedy J: Changes in herb and dietary supplement use in the US adult population: a comparison of the 2002 and 2007 National Health Surveys. Clin Ther 2011, 33(11):1749-1758.

82. Massad SJ, Shier NW, Koceja DM, Ellis NT: High school athletes and nutritional supplements: a study of knowledge and use. Int J Sports Nutr 1995, 5(3):232-245.

83. Froiland K, Koszewski W, Hingst J, Kopecky L: Nutrition supplement use among college athletes and their sources of information. Int I Sports Nutr Exerc Metabol 2004, 14:104-120.

84. Fitness CONCOSMFCoNatCoSMa: Sport drinks and energy drinks for children and adolescents: are they appropriate? Pediatrics 2011, 127:1182-1189.

85. Popkin BM, Armstrong LE, Bray GM, Caballero B, Frei B, Willett WC: A new proposed guidance system for beverage consumption in the United States. Am J Clin Nutr 2006, 83:529-542.

86. Lun V, Erdman KA, Fung TS, Reimer RA: Dietary supplementation practices in Canadian high-performance athletes. Int J Sports Nutr Exerc Metabol 2012, 22:31-37.

87. Braun H, Koehler K, Geyer H, Kleinert J, Mester J, Schanzer W: Dietary supplement use among elite young German athletes. Int J Sport Nutr Exerc Metab 2009, 19:97-109.

88. Baylis A, Cameron-Smith D, Burke LM: Inadvertent doping through supplement use by athletes: assessment and management of the risk in Australia. Int $J$ Sport Nutr Exerc Metab 2001, 11:365-383.

89. Park S, Onufrak S, Blanck HM, Sherry B: Characteristics associated with consumption of sports and energy drinks among US adults: National Health Interview Survey, 2010. J Acad Nutr Diet 2013, 113:112-119.

90. Han E, Powell LM: Consumption patterns of sugar sweetened beverages in the United States. J Acad Nutr Diet 2013, 113:43-53.

91. Greenwood M, Ferris J, Kreider R, Greenwood L, Byars A: Creatine supplementation patterns and perceived effects in select Division I collegiate athletes. Clin J Sport Med 2000, 10:191-194.

92. LaBotz M, Smith BW: Creatine supplement use in an NCAA Division I athletic program. Clin J Sport Med 1999, 9:167-169.

93. Rosen O, Sundgot-Borgen J, Maehlum S: Supplement use and nutritional habits in Norwegian elite athletes. Scand J Med Sci Sports 1999, 9:28-35.

94. Scofield DE, Unruh S: Dietary supplement use among adolescent athletes in Central Nebraska and their sources of information. J Strength Cond Res 2006, 20(2):452-455.

95. Birch $R$, Nobel D, Greenhaff PL: The influence of dietary creatine supplementation on performance during repeated bouts of maximal isokinetic cycling in man. Eur J Appl Physiol Occ Physiol 1994, 69:268-270.

96. Casey A, Constantin-Teodosiu D, Howell S, Hultman E, Greenhaff PL: Creatine ingestion affects performance and muscle metabolism during maximal exercise in humans. Am J Physiol Endocrinol Metabol 1996, 271:E31-E37.
97. Balsom PD, Ekblom B, Soderlund K, Sjodin B, Hultman E: Creatine supplementation and dynamic high-intensity intermittent exercise. Scand J Med Sci Sports 1993, 3:143-149.

98. Gotshalk LA, Volk JS, Staron RS, Denegar CR, Hagerman FC, Kraemer WJ: Creatine supplementation improves muscular performance in older men. Med Sci Sports Exerc 2002, 34(3):537-543.

99. Vandenberghe K, Goris M, VanHecke P, Leemputte MV, VanGerven L, Hespel P: Long-term creatine intake is beneficial to muscle performance during resistance exercise. J App/ Physiol 1997, 83(6):2055-2063.

100. Istitutes of Medicine: Dietary reference intakes for energy, carbohydrates, fiber, fat, fatty acids, cholesterol, protein, and amino acids. A report of the Panel on Macronutrients, Subcommittee on Upper Levels of Nutrients and Interpretation and Uses of Dietary Reference Intake, and the Standing Committee on the Scientific Evaluation of Dietary Reference Intakes. Washington DC: National Academies Press; 2005.

101. Phillips SM: Protein requirements and supplementation in strength sports. Nutrition 2004, 20:689-695.

102. Tarnopolsky M: Protein requirements for endurance athletes. Nutrition 2004, 20:662-668.

103. Pasiakos SM, Austin KG, Lieberman HR, Askew EW: Efficacy and safety of protein supplements for US Armed Forces personnel: consensus statement. J Nutr 2013, 143(11):1811S-1814S.

104. Cermak NM, Res PT, deGroot LCPGM, Saris WHM, vanLoon LJC: Protein supplementation augments the adaptive response of skeletal muscle to resistance-type exercise training: a meta-analysis. Am J Clin Nutr 2012, 96:1454-1464

105. Reissig CJ, Strain EC, Griffiths RR: Caffeinated energy drinks-a growing problem. Drug Alcohol Depend 2009, 99:1-10.

106. Duchan E, Patel ND, Feucht C: Energy drinks: a review of use and safety for athletes. Physician Sportsmed 2010, 38(2):171-179.

107. McLellan TM, Lieberman HR: Do energy drinks contain active compounds other than caffeine? Nutr Rev 2012, 70(12):730-744.

108. Seifert SM, Schaechter JL, Hershorin ER, Lipshultz SE: Health effects of energy drinks on children, adolescents, and young adults. Pediatrics 2011, 127(3):511-528.

109. Astorino TA, Roberson DW: Efficacy of acute caffeine ingestion for shortterm high-intensity exercise performance: a systematic review. J Strength Cond Res 2010, 24(1):257-265

110. Davis JK, Green JM: Caffeine and anaerobic performance. Ergogenic value and mechanisms of action. Sports Med 2009, 39(10):815-852

111. Graham TE: Caffeine and exercise. Metabolism, endurance and performance. Sports Med 2001, 31(11):785-807.

112. Keisler BD, Armsey TD: Caffeine as an ergogenic aid. Curr Sports Med Rep 2006, 5:215-219.

113. Krumbach CJ, Ellis DR, Driskell JA: A report on vitamin and mineral supplement use among university athletes in a division I institution. Int J Sport Nutr 1999, 9:416-425

114. Petroczi A, Naughton DP, Mazanov J, Holloway A, Bigham J: Performance enhancement with supplements: incongruence between rationale and practice. J Int Soc Sports Nutr 2007, 4:19.

115. Kristiansen M, Levy-Milne R, Barr S, Flint A: Dietary supplement use by varsity athletes at a Canadian university. Int I Sports Nutr Exerc Metabol 2005, 15:195-210.

doi:10.1186/1472-6882-14-143

Cite this article as: Knapik et al:: A systematic review and meta-analysis on the prevalence of dietary supplement use by military personnel. BMC Complementary and Alternative Medicine 2014 14:143. 